\title{
Near-Earth Magnetic Field Effects of Large-Scale Magnetospheric Currents
}

\author{
Lühr, Hermann; Xiong, Chao; Olsen, Nils; Le , Guan
}

Published in:

Space Science Reviews

Link to article, DOI:

10.1007/s11214-016-0267-y

Publication date:

2017

Document Version

Peer reviewed version

Link back to DTU Orbit

Citation (APA):

Lühr, H., Xiong, C., Olsen, N., \& Le , G. (2017). Near-Earth Magnetic Field Effects of Large-Scale

Magnetospheric Currents. Space Science Reviews, 206, 521-545. https://doi.org/10.1007/s11214-016-0267-y

\section{General rights}

Copyright and moral rights for the publications made accessible in the public portal are retained by the authors and/or other copyright owners and it is a condition of accessing publications that users recognise and abide by the legal requirements associated with these rights.

- Users may download and print one copy of any publication from the public portal for the purpose of private study or research.

- You may not further distribute the material or use it for any profit-making activity or commercial gain

- You may freely distribute the URL identifying the publication in the public portal

If you believe that this document breaches copyright please contact us providing details, and we will remove access to the work immediately and investigate your claim. 
Revised version to Space Science Review for Special Issue "Earth's Magnetic Field" Near-Earth magnetic field effects of large-scale magnetospheric currents

Hermann Lühr ${ }^{1)}$, Chao Xiong ${ }^{1)}$, Nils Olsen ${ }^{2)}$, and Guan $\mathrm{Le}^{3)}$

1) GFZ, German Research Centre for Geosciences, Section 2.3, Earth's Magnetic Field, Potsdam, Germany

2) DTU Space, National Space Institute, Technical University of Denmark, Lyngby, Denmark

3) NASA Goddard Space Flight Center, Heliophysics Science Division, Greenbelt, Maryland, USA

\begin{abstract}
Magnetospheric currents play an important role in the electrodynamics of nearEarth space. This has been the topic of many space science studies. Here we focus on the magnetic field they cause close to Earth. Their contribution to the geomagnetic field is the second largest after the core field. Significant progress in interpreting the magnetic fields from the different sources has been achieved thanks to magnetic satellite missions like Ørsted, CHAMP and now Swarm. Of particular interest for this article is a proper representation of the magnetospheric ring current effect. Uncertainties in modelling its effect still produce the largest residuals between observations and present-day geomagnetic field models. A lot of progress has been achieved so far, but there are still open issues like the characteristics of the partial ring current. Other currents discussed are those flowing in the magnetospheric tail. Also their magnetic contribution at LEO orbits is non-negligible. Treating them as an independent source is a more recent development, which has cured some of the problems in geomagnetic field modelling. Unfortunately there is no index available for characterising the tail current intensity. Here we propose an approach that may help to properly quantify the magnetic contribution from the tail current for geomagnetic field modelling. Some open questions that require further investigation are mentioned at the end.
\end{abstract}


Keywords Geomagnetic field, Magnetospheric currents, Magnetospheric ring current, Magnetospheric tail currents, Geomagnetic field modelling

\section{Introduction}

The geomagnetic field as observed on ground or by low-Earth orbiting (LEO) satellites is the sum of contributions from many different sources. The largest part, the core field accounting for more than $90 \%$, originates from dynamo action in the Earth's fluid outer core. Another internal source is the magnetisation of rocks and sediments at depths up to, say, $20 \mathrm{~km}$, comprising the "lithospheric field". Magnetic fields, generated by electric currents in the ionosphere and magnetosphere, are termed external sources. These magnetic fields are highly variable in time and space. As a consequence, they induce electric currents in the electrically conducting subsurface layers of Earth; their resulting magnetic fields are called induction fields. In this chapter we will repeatedly refer to these terms when discussing the different contributions.

Magnetic field observations have successfully been used in the past for remotely sensing physical processes related to the source mechanisms of the different components. However, a prerequisite for applying such a technique is a proper separation of the various field contributions and an isolation of the signal of interest. This is still a challenging task and further improvements are warranted for a full utilisation. A typical approach for the separation of source terms is to consider their differences in characteristics both in time and space. As expected, there are overlaps in the characteristic between the different source terms, and therefore no simple techniques are available for a clear source separation.

In recent years the quality of geomagnetic field models has improved considerably. This is in first place due to the high quality of magnetic measurements provided by dedicated satellite 
missions like Ørsted and CHAMP and now also the Swarm constellation of three satellites. In addition, also the techniques for field modelling have evolved a lot during the past decade. A first and important step is a clever scheme for selecting those magnetic field data, which are not so strongly influenced by contributions from external sources. On the other hand, a sufficient amount of data has to be taken into account in order to achieve statistically relevant results.

In this article we will focus on the magnetic fields generated by magnetospheric currents. Their effect is the second largest in the concert of contributions, as observed at near-Earth locations, outside the auroral oval. For that reason a proper description of their source terms is of special importance for the numerical modelling of other geomagnetic field parts. Within the magnetosphere there are several major current systems. These include the ChapmanFerraro currents on the dayside magnetopause, the magneto-tail currents on the nightside, the magnetospheric ring current in the equatorial plane at a typical distant of about 5 Earth radii $\left(\mathrm{R}_{\mathrm{E}}\right)$ and the field-aligned currents (FACs) connecting magnetospheric currents with the ionosphere at auroral latitudes. A schematic illustration of major magnetospheric current systems is shown in Figure 1. Different colours have been used for highlighting the various current systems. More details about the characteristics of these currents can be found in text books like Kivelson and Russell (1995).

The purpose of this article is to assess the influence of large-scale magnetospheric current systems on attempts to model the core and lithospheric field. Certain approaches have been developed to minimise the effects of external field contributions, but these are still not sufficient. Unmodelled external field contributions pose presently the largest problems for progress in geomagnetic field modelling. This is partly due to the imperfectness of geomagnetic activity indices like $\mathrm{Kp}, \mathrm{D}_{\mathrm{ST}}$ or $\mathrm{AE}$. For some magnetospheric and ionospheric current systems suitable proxies for quantifying their intensity are completely missing. Here 
we are going to present some alternatives that may help to improve the situation. Our investigations are based on data from globally distributed ground magnetic observatories and from the satellites Ørsted, CHAMP and Swarm.

In the sections to follow we will first present general features of the main magnetospheric currents and introduce proxies for quantifying them. Subsequently detailed descriptions of the ring current and magnetospheric tail current will follow. Special attention will be paid to possibilities of parameterising the near-Earth magnetic field effects of these currents. Our prime aim is to outline an improved approach for considering the external field contributions for geomagnetic field modelling.

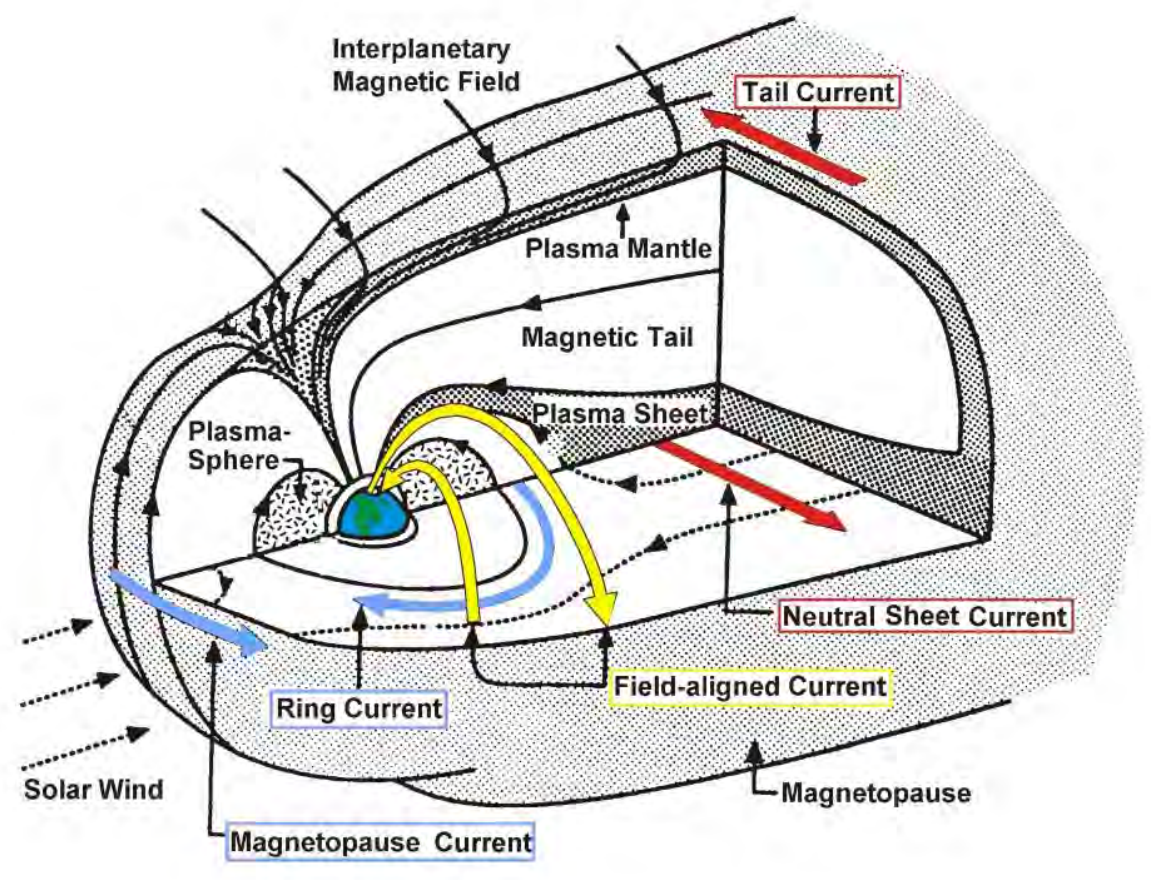

Fig. 1 Schematic illustration of magnetospheric current systems contributing to the nearEarth magnetic field. The major current systems are highlighted by different colours. (modified after Fig. of Kilvelson and Russell, 1995). 


\section{General features of magnetospheric currents}

For describing the activity of the various magnetospheric currents it is advisable to use appropriate coordinate systems. Since the solar wind is the prime driver for magnetospheric activity, the direction to the sun plays a central role for the geometry of the currents. Furthermore, the Earth's internal magnetic field acts as the reference frame for the dynamics of charged particles. In situ observations of the ring current have shown that the geomagnetic main field (primarily its dipole terms) closely controls the latitudinal current distribution (see Hamilton et al. (1986) and references therein). For that reason it is good practice to present the magnetic effect of the ring current in Solar-Magnetic (SM) coordinates. In that system the $\mathrm{z}$ axis is aligned with the geomagnetic dipole axis, pointing northward, the $\mathrm{y}$ axis is perpendicular to the plane spanned by the dipole axis and the direction to the sun, pointing toward the evening side, and the $\mathrm{x}$ axis completes the triad, pointing towards the sun. In case of a symmetric ring current the magnetic effect at Earth is aligned with the SM z component. Since westward currents are dominating in the ring current, generally negative SM z values are observed.

Currents flowing further away from Earth, in regions where the main field is weaker, are more closely controlled by the influence of the solar wind. Their effect is best described in Geocentric-Solar-Magnetospheric (GSM) coordinates. In that frame the $\mathrm{x}$ axis is pointing from the Earth to the sun, the y axis is perpendicular to the plane containing the geomagnetic dipole axis and the $\mathrm{x}$ axis, pointing towards the evening side, and the $\mathrm{z}$ axis completes the triad pointing northward. The two systems, SM and GSM, are rather closely aligned. Therefore magnetic contributions in these frames cannot easily be separated. Largest angles between the two systems occur during solstice seasons and smallest during equinoxes. A more detailed introduction into these and other space physics related coordinate systems can be found in the Appendix of Kivelson and Russell (1995, pg. 531ff). 
The geometry of the ring current is strongly controlled by the Earth's main field. Therefore its magnetic effect at Earth generally has a spatial distribution fixed in latitude and longitude. Just the amplitude changes with time but not the distribution. Exceptions occur during magnetic storms when a partial ring current develops. Such phenomena will be discussed in more detail in Section 3.2.

The situation is quite different for the effect of magneto-tail currents. The orientation and location of these currents is controlled by a combined action of the main field and the solar wind. At a fixed location on Earth the tail currents produce a time varying magnetic field comprising both diurnal and annual variations. The amplitudes of these variations observed in the three field components depend on latitude, longitude and season, with largest values during the solstices. Further details of the tail current effects are given in Section 4.1.

Despite of dedicated satellite missions like Cluster, THEMIS and MMS there is still no continuous in situ monitoring of magnetospheric currents. Indirect quantities that can be used to quantify their intensity are therefore desirable. In case of the ring current the $\mathrm{D}_{\mathrm{ST}}$ (disturbance storm time) index, introduced in the 1960s (Sugiura, 1964), is commonly used for this purpose. It reflects the longitudinal mean magnetic disturbance in nano Tesla $[\mathrm{nT}]$ at the dipole equator caused by the magnetospheric ring current. In practice the $\mathrm{D}_{\mathrm{ST}}$ value is derived from four magnetic observatories at mid latitudes, separated approximately by $90^{\circ}$ in longitude. More details on the techniques for actually deriving the $\mathrm{D}_{\mathrm{ST}}$ index can be found in (Iyemori, 1990). The $\mathrm{D}_{\mathrm{ST}}$ index is commonly used to quantify the intensity of a geomagnetic storm, the larger the negative deflections the stronger the storm.

The situation is less favourable for the currents in the outer magnetosphere. No index exists that can be used to quantify their activity. It is known that the Chapman-Ferraro currents on the dayside magnetopause get stronger when the solar wind dynamic pressure increases. They generate a magnetic field that compensates the main field at locations outside the 
magnetosphere. The more the boundary is pushed towards the Earth, the stronger this shielding field. Inside the magnetosphere the field of the Chapman-Ferraro currents are enforcing the main field. Since the shape of the dayside magnetopause is reasonably well known, the magnetic effect of the Chapman-Ferraro currents can be estimated quite reliably when the solar wind pressure is known from in situ measurements. In addition the interplanetary magnetic field has to be taken into account. In case of a southward component the size of the magnetosphere can be further reduced (e.g. Sibeck et al., 1991). All these effects are taken care of by present-day magnetopause models (e.g. Shue et al., 1998).

Another relevant contribution of magnetic field comes from the magnetospheric tail current system. As can be seen in Figure 1, the neutral sheet current flows westward in the central plane of the tail. It diverts at the magnetopause; half of it is flowing over the northern tail lobe and the other half over the southern lobe, closing the cross-tail current loop. At times of magnetic reconnection between the interplanetary magnetic field (IMF) and Earth's main field, additional magnetic flux is stored in the magneto-tail (e.g. Hughes, 1995). Favourable conditions for an enlarged tail exist when the IMF has a southward component, opposite to the direction of the geomagnetic field. Then closed magnetic field lines will be opened on the dayside through magnetic reconnection and transported tailward by the solar wind. As a consequence of the increased magnetic flux in the tail the neutral sheet current gets stronger and an enhanced southward-directed magnetic contribution is observed close to Earth. Unfortunately, there is presently no index available that reliably quantifies the intensity of the magneto-tail currents.

Further phenomena of interest are magnetospheric substorms. Under certain conditions, after excessive loading, magnetic energy stored in the magneto-tail is released explosively. Part of the energy is convected downtail, but the other part is routed towards the Earth along magnetic field lines into the auroral regions. According to our present understanding, the 
neutral sheet cross-tail current is partly disrupted and rerouted along field lines through the auroral ionosphere (e.g. Clauer and McPherron, 1974; Ritter and Lühr, 2008). This reduced cross-tail current causes an enhancement of the magnetic field at the time of substorm onset. The effect fades away after about two hours. Occurrences of substorms can reliably be detected at auroral latitudes where they make large effects. In a statistical study Ritter and Lühr (2008) have investigated the magnetic effect of substroms at mid and low latitudes. Enhancements of the northward component of more than $10 \mathrm{nT}$ have been observed. But for substorms occurring during quiet times $\left(K p<=2^{\circ}\right)$, outside magnetic storms, the low and mid latitude effects hardly exceeds $2 \mathrm{nT}$ and thus have only marginal influence on geomagnetic field modelling.

\section{The magnetospheric ring current and its representation}

The magnetospheric ring current has a "doughnut-like" shape encircling Earth near the equatorial plane at distances from 2 to $7 \mathrm{R}_{\mathrm{E}}$. Currents are carried by charged particles trapped by the geomagnetic field. Ions drift westward in the main part of the ring current while the electrons move eastward, resulting in a net westward current. During magnetic storms or substorms more energetic charged particles are injected and the ring current becomes stronger and moves closer to Earth. When the supply of particles stops, the current intensity gradually decays, which is termed the recovery phase of the storm. Because of the close relationship between storm evolution and ring current intensity, the corresponding $\mathrm{D}_{\mathrm{ST}}$ index is commonly used as storm-time indicator.

An electric current at several $\mathrm{R}_{\mathrm{E}}$ distance, like the ring current centred in the magnetic equator plane, provides at Earth a uniform magnetic field aligned with the magnetic dipole axis and pointing southward. Such a field appears in the magnetic field measurements of polar orbiting 
satellites as the external $\mathrm{q}_{1}{ }^{0}$ term in spherical harmonic expansions. The advantage of satellite measurements over the ground-based recordings is that they sense the absolute amplitude. Data from ground observatories track well the temporal variations, but cannot determine an unknown bias value (e.g. Langel et al., 1980; Langel and Estes, 1985) because of local (unknown) lithospheric field contributions in the vicinity of the observatory. There is always a ring current flowing, also during quiet times when the $\mathrm{D}_{\mathrm{ST}}$ index is zero.

In the subsequent sections we will first introduce properties of the quiet-time ring current, in particular its representation by indices. Thereafter we address features of the partial ring current that appears during magnetically disturbed periods. Different types of observations have so far not been able to provide a unified picture of the actual current geometry.

\subsection{The quiet-time ring current}

In this section we focus on the characteristics of external fields during quiet times, which is different from many other space science studies. Satellite data are used to check the reliability of the $\mathrm{D}_{\mathrm{ST}}$ index or equivalent parameters. Lühr and Maus (2010) reported about systematic ring current measurements over 9 years with the CHAMP satellite. Only data from quiet times have been taken, when the magnetic activity index $a_{p}$ was below 15 (corresponding to $K p<$ $\left.3^{0}\right)$ and in addition the previous 3-hour interval satisfied $a_{p}<18\left(K p<3^{+}\right)$. The obtained results therefore do not represent the average ring current activity during those years.

Spherical harmonic analysis allows to separate, in case globally distributed observations are available, between magnetic field contributions originating from sources inside and outside the orbital altitude of the satellite. In this way we have determined the external, magnetospheric, field contributions in the satellite data. Experience has shown that it makes sense to further separate the external fields, by means of spherical harmonic analysis, into 
their parts that are better ordered in the SM and GSM frames (e.g. Maus and Lühr, 2005). Magnetic data of at least one year are needed for distinguishing reliably between the average field contributions in the two frames. Here we focus on the SM part, which is related to the ring current.

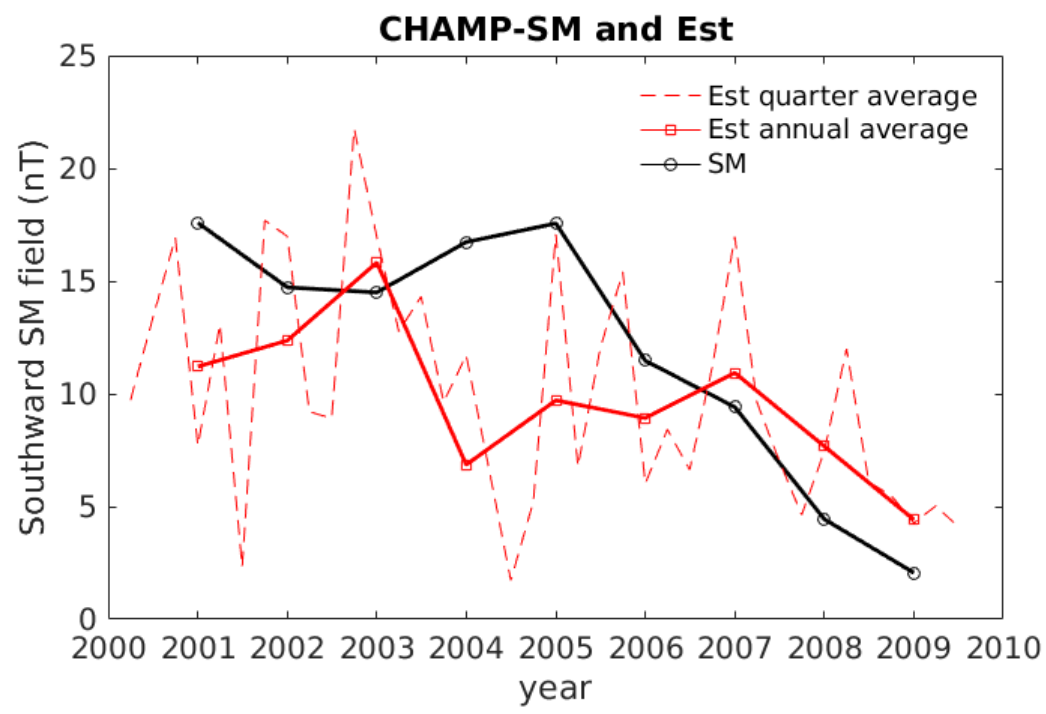

Fig. 2 Quiet-time ring current index. Annual average of the ring current field as derived by CHAMP (black curve). External part of $\mathrm{D}_{\mathrm{ST}}$ index, $\mathrm{E}_{\mathrm{ST}}$, is shown by red curves, as annual averages (solid line) and 3-month averages (dashed line).

Figure 2 shows the obtained CHAMP results of the SM $-\mathrm{z}$ component for the years from 2001 through 2009 as black curve. During the active years of solar cycle 23, up to 2005, annual averages beyond $15 \mathrm{nT}$ are reached. Thereafter values gradually decline approaching $2 \mathrm{nT}$ in 2009. This solar cycle dependence is present although only quiet intervals (according to Kp) have been considered. Obviously, intervals between magnetically active periods are too short during solar maximum years for the ring current to fully decay. 
These satellite-based results cannot be compared directly with $\mathrm{D}_{\mathrm{ST}}$ values. The ground-based magnetic field measurements used to derive $\mathrm{D}_{\mathrm{ST}}$ are the sum of the external part, caused by the magnetospheric currents and the internal part from the corresponding induction effect. Maus and Weidelt (2004) and Olsen et al. (2005) have proposed an approach for decomposing $D s t=E s t+I s t$ into its external part $\mathrm{E}_{\mathrm{ST}}$ and the internal part $\mathrm{I}_{\mathrm{ST}}$, caused by ground induced currents, with

$$
\operatorname{Ist}(t)=Q \operatorname{Est}(t)
$$

The factor $Q$ is determined from radially symmetric profiles of electrical conductivity in the Earth's mantle. A typical value is $Q=0.28$; for a more realistic mantle profile with non-zero but finite conductivity the multiplication of (1) has to be replaced by a convolution.

Nowadays the decomposition of $\mathrm{D}_{\mathrm{ST}}$ into $\mathrm{E}_{\mathrm{ST}}$ and $\mathrm{I}_{\mathrm{ST}}$ is done routinely and the two parts are considered separately in geomagnetic field modelling. When correcting the field components X, Y, Z (northward, eastward, downward, respectively) of an observatory for the ring current effect, $\mathrm{E}_{\mathrm{ST}}$ and $\mathrm{I}_{\mathrm{ST}}$ are to be used

$$
\begin{aligned}
& X^{\prime}=X-E s t \cos (\beta)-I s t \cos (\beta) \\
& Z^{\prime}=Z-E s t \sin (\beta)+2 \text { Ist } \sin (\beta)
\end{aligned}
$$

where $X^{\prime}$ and $Z^{\prime}$ are the corrected northward and downward components, respectively, $\beta$ is the dipole latitude of the observatory. The Y component generally does not need correction.

For our comparison of the $\mathrm{D}_{\mathrm{ST}}$ index with the CHAMP SM annual averages we have to consider only the $\mathrm{E}_{\mathrm{ST}}$ values. Seasonal and annual averages of $\mathrm{E}_{\mathrm{ST}}$ have been calculated by using the same selection criteria as for CHAMP data. The evolution of $\mathrm{E}_{\mathrm{ST}}$ is plotted in Figure 2 as red lines. The dashed red line reflects clearly the seasonal variation of magnetic activity. Minima appear commonly around June solstice and maxima are observed during equinox 
and/or December solstice months. The June solstice depression is a well-known characteristic of the upper atmosphere clearly visible in air density, electron density and also magnetic activity. The annual averages of $\mathrm{E}_{\mathrm{ST}}$ (solid red line) follow to some extent the activity level of the solar cycle, but the differences to the CHAMP SM results are substantial, reaching values up to $10 \mathrm{nT}$ during the active phase of the solar cycle. Differences become smaller around the minimum phase. It is interesting to note that all these activity-dependent features are visible in the averages although only values from quiet times $(K p<3)$ have been considered.

These obvious deficits of the $\mathrm{D}_{\mathrm{ST}}$ index have initiated activities for an improved representation of the ring current activity. An alternative ring current index, called $\mathrm{RC}$, is derived from 21 globally distributed magnetic observatories at mid and low latitudes (not at the magnetic equator, to avoid contributions from the equatorial electrojet). As described by Olsen et al. (2014), a core field magnetic model, like CHAOS-4, is subtracted from the ground observatory hourly mean values. Remaining crustal magnetic field biases are determined and subsequently removed individually for each station. The crustal bias is a constant value, the arithmetic mean of quiet-time $\left(K p<2^{+}\right)$night values over the whole time span ( $>10$ years) is in consideration. A spherical harmonic analysis is applied to the residuals of the magnetic northward components from the considered observatories. For this hour-byhour analysis only stations in darkness $(18-06$ LT) are given account, and their location is taken in dipole coordinates. The $\mathrm{RC}$ index is derived from the central external dipole term (e.g. $-\mathrm{q}_{1}{ }^{0}$ ) of the analysis. It is thus compatible with the (external part of the) $\mathrm{D}_{\mathrm{ST}}$ index as the southward deflection of the magnetic field at the geomagnetic equator.

According to the arguments made above, also RC has to be separated into the external and internal parts, ERC and IRC, respectively. For the comparison with CHAMP SM annual averages only ERC is of interest. 
It should be noted that there are also other attempt for representing external field contributions, e.g. by the VMD index, specifically designed for main field modelling (Thompson and Lesur, 2007).

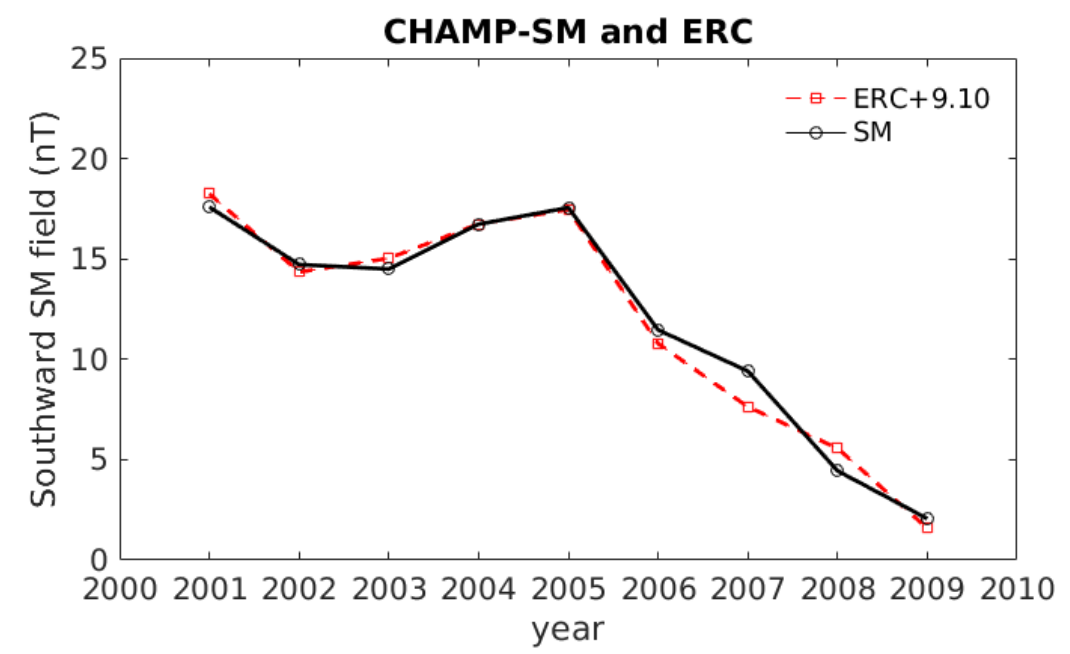

Fig. 4 (black curve) Annual averages of CHAMP SM values (same as Fig. 3). (red curve) External part of RC index, ERC, also annual averages. The red curve has been offset by 9.1 nT for best fitting the CHAMP values.

Figure 4 shows the quiet-time ring current effect, independently determined from ground stations and from CHAMP over 9 years. We find an almost perfect match between the two sets of annual averages. There is just a constant bias of $9.1 \mathrm{nT}$ by which the ERC value has to be made more negative (accounting for the unknown constant bias of RC) to properly reflect the ring current activity. By comparing Figures 3 and 4 we can seen that satellites can track the quiet-time ring current effect very well. Furthermore, the RC index is much more consistent with the results derived by CHAMP than the $\mathrm{D}_{\mathrm{ST}}$ index. For the geomagnetic field modelling the ring current effect has to be estimated hour by hour. This can best be achieved by ground-based observations. Our results suggest that the $\mathrm{RC}$ index is a suitable parameter for providing that information. For completeness it should be mentioned that satellite-derived 
main field models have been used for determining the baselines of the observatory readings. By this procedure a certain amount of signal feed-through may have helped to get the good fit between the ERC and the CHAMP-SM in Figure 4.

\subsection{The asymmetric ring current}

According to the standard procedure, the $\mathrm{D}_{\mathrm{ST}}$ value represents the longitudinal average of the northward magnetic disturbance at low latitudes. This assumes that the ring current is an azimuthally symmetric current. However, it is known since long (e.g. Akasofu and Chapman, 1964) that magnetic deflections are stronger in certain local time sectors during storm times than in others. This effect has been termed the asymmetric ring current. As $\mathrm{D}_{\mathrm{ST}}$ is not capable of reflecting the asymmetry, additional indices have been developed by the University of Kyoto's World Data Center, such as ASY-H and ASY-D (Iyemori, 1990) that reflect the maximum longitudinal differences in the northward and eastward components, respectively. So far these have not been endorsed by IAGA.

An alternative way to measuring the magnetic effect of the ring current by ground stations can be provided by LEO satellites on near-equatorial orbits. They provide a full longitudinal scan on every orbit ( $\sim 95 \mathrm{~min})$. An example for that is the US Air Force satellite C/NOFS (de La Beaujardière, et al., 2004), which was launched in April 2008 and re-entered in November 2015. With its orbital inclination of only $13^{\circ}$ it stays within a latitudinal distance of $\pm 24^{\circ}$ from the geomagnetic equator. This is a favourable orbit for detecting magnetic effects of the ring current. As part of its space science instrument suit C/NOFS carried also a vector magnetometer. Magnetometer data have been calibrated with respect to high-quality geomagnetic field models, POMME-6 in the beginning and POMME-8 for later years, mainly based on CHAMP data. Magnetic field readings from C/NOFS of the years from 2008 
through 2010 have been considered by Le et al. (2011) to study the ring current evolution during storms. For isolating the ring current effect first the core and crustal fields as given by the models POMME-6 and MF7, respectively, are removed from the satellite magnetic field readings. The residuals are transformed into the SM system and only the SM z component is used where the major effect is expected. Close to the magnetic equator the equatorial electrojet (EEJ) may also contribute to a negative magnetic deflections by about $10 \mathrm{nT}$ around local noon.

Examples of direct comparison between C/NOFS observations and $\mathrm{D}_{\mathrm{ST}}$ values are presented in Figure 5 for the storm on 22 July 2009. The top panel presents the evolution of the moderate storm with a peak $\mathrm{D}_{\mathrm{ST}}$ value of only $-80 \mathrm{nT}$. It starts early on $22 \mathrm{July}$, and the main phase lasts for about 4 hours. At representative epochs, marked by numbered vertical lines, the ring current effects are determined. In the lower part C/NOFS results, $\mathrm{C}_{\mathrm{ST}}$, for these epochs are shown as black line in a dial plot as the satellite scans through all local times over one orbit. For a better visualisation the readings are offset by $100 \mathrm{nT}$. The plotted values are thus

$$
C_{S T}=-z_{S M}+100 n T
$$

For quantifying the $\mathrm{C} / \mathrm{NOFS}$ results a red circle is fitted to the $\mathrm{C}_{\mathrm{ST}}$ values sampled over an orbit. Parameters derived from this fit are the radius and the position of centre in terms of both its offset and local time. The offset quantifies the longitudinal asymmetry of the ring current, and the local time marks the sector of largest enhancement. In order to reduce the influence of the EEJ we omitted readings in the circle fit when their magnetic latitude is within $\pm 5^{\circ}$ MLat and their local time is within the sector $08-16$ LT. Accordingly the corresponding $\mathrm{D}_{\mathrm{ST}}$ value is plotted as blue symmetrical ring also offset by $-100 \mathrm{nT}$. The bias prevents a collapse of all data in the centre for small values and allows to present positive-field ring current effects. 


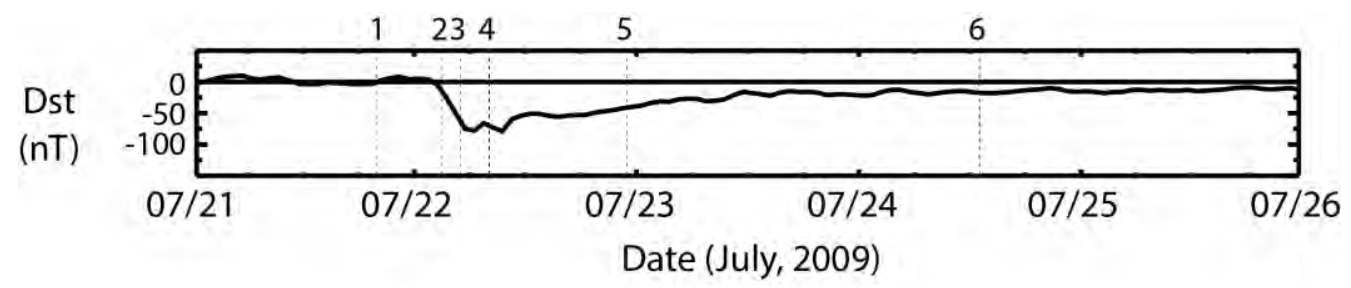

(1) 2000 UT, 2009-07-21

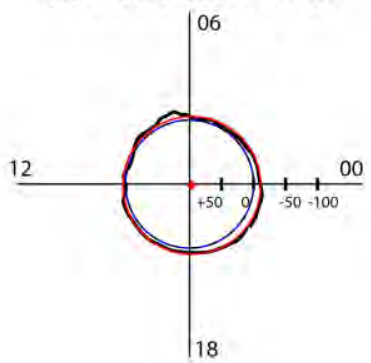

(4) 08:00 UT, 2009-07-22

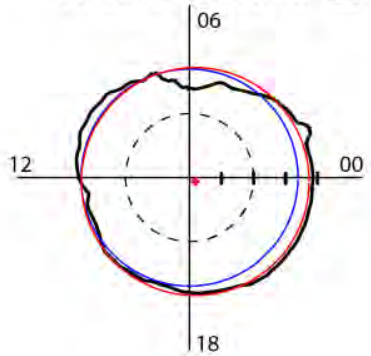

(2) 0300 UT, 2009-07-22

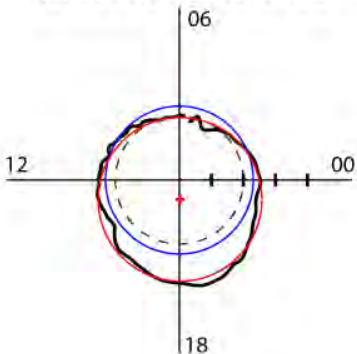

(5) 2300 UT, 2009-07-22

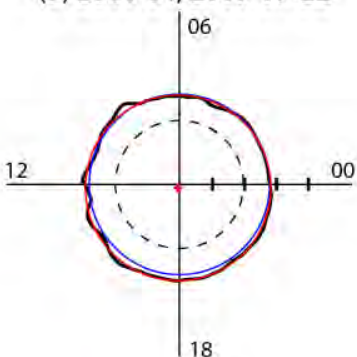

(3) 0500 UT, 2009-07-22



(6) 1300 UT, 2009-07-24

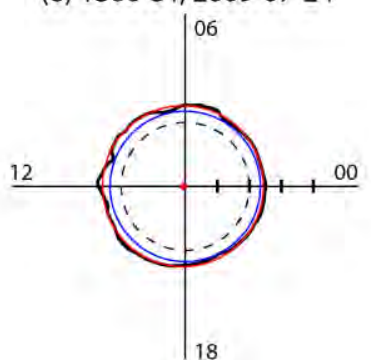

Fig. 5 Comparison of the magnetic ring current effect measurements by C/NOFS ( $\left.\mathrm{z}_{\mathrm{SM}}\right)$ near the equatorial plane (black curve) with $\mathrm{D}_{\mathrm{ST}}$ values (blue circle) during 4 days around a magnetic storm. Red circles are fits to C/NOFS observations and red dots mark their centres. Dashed circles are the reference for $\mathrm{D}_{\mathrm{ST}}=0$ (after Fig. 3 of Le et al., 2011).

Figure 5 covers 5 days of $\mathrm{D}_{\mathrm{ST}}$ evolution around the magnetic storm. Several hours before the onset there is a quiet magnetic field (upper left dial). C/NOFS observes a nicely circular and centred field distribution. Only one hour into the main phase (upper middle dial) the magnetic effect increases predominantly around the evening sector. As a consequence the centre of fitted circle is displaced by about $30 \mathrm{nT}$ towards the $18 \mathrm{LT}$ sector. By that time $\mathrm{D}_{\mathrm{ST}}$ starts to 
underestimate the disturbance. Two hours later (upper right dial), the storm has further intensified and C/NOFS records an even stronger asymmetry of more than $50 \mathrm{nT}$, now shifted somewhat to later evening. $\mathrm{D}_{\mathrm{ST}}$ clearly underestimates the mean disturbance level and by definition cannot reflect the asymmetry. About an hour after completion of main phase (bottom left dial) C/NOFS finds again a well-centred deflection pattern at all longitudes, and $\mathrm{D}_{\mathrm{ST}}$ agrees reasonably well with $\mathrm{C} / \mathrm{NOFS}$ recordings. This fair match between satellite observation and index of the symmetric ring current distribution continues through the recovery phase of the storm (bottom middle and right dials).

This one example indicates that generally the ring current effect is evenly distributed through all longitudes (local times), and the representation by a single number like $\mathrm{D}_{\mathrm{ST}}$ is justified. However, during the main phase of a magnetic storm a significantly asymmetric magnetic deflection is found around the globe, and $\mathrm{D}_{\mathrm{ST}}$ typically underestimates the peak deflection. Le et al. (2011) studied four individual storms and confirmed similar ring current features in all the cases. In order to check the general validity of the statements on asymmetry we considered a large numbers of C/NOFS orbits independent of magnetic activity covering the years from 2009 through 2013. For each orbit a circle was fitted to the C/NOFS readings and the centre point was determined. Figure 6 shows the positions of centre points in a dial plot. Results are sorted into four classes of magnetic activity, $K p$ : 0-2, 2-4, 4-6, >6. Individual centres are marked by black dots and a red dot represents the median position. 

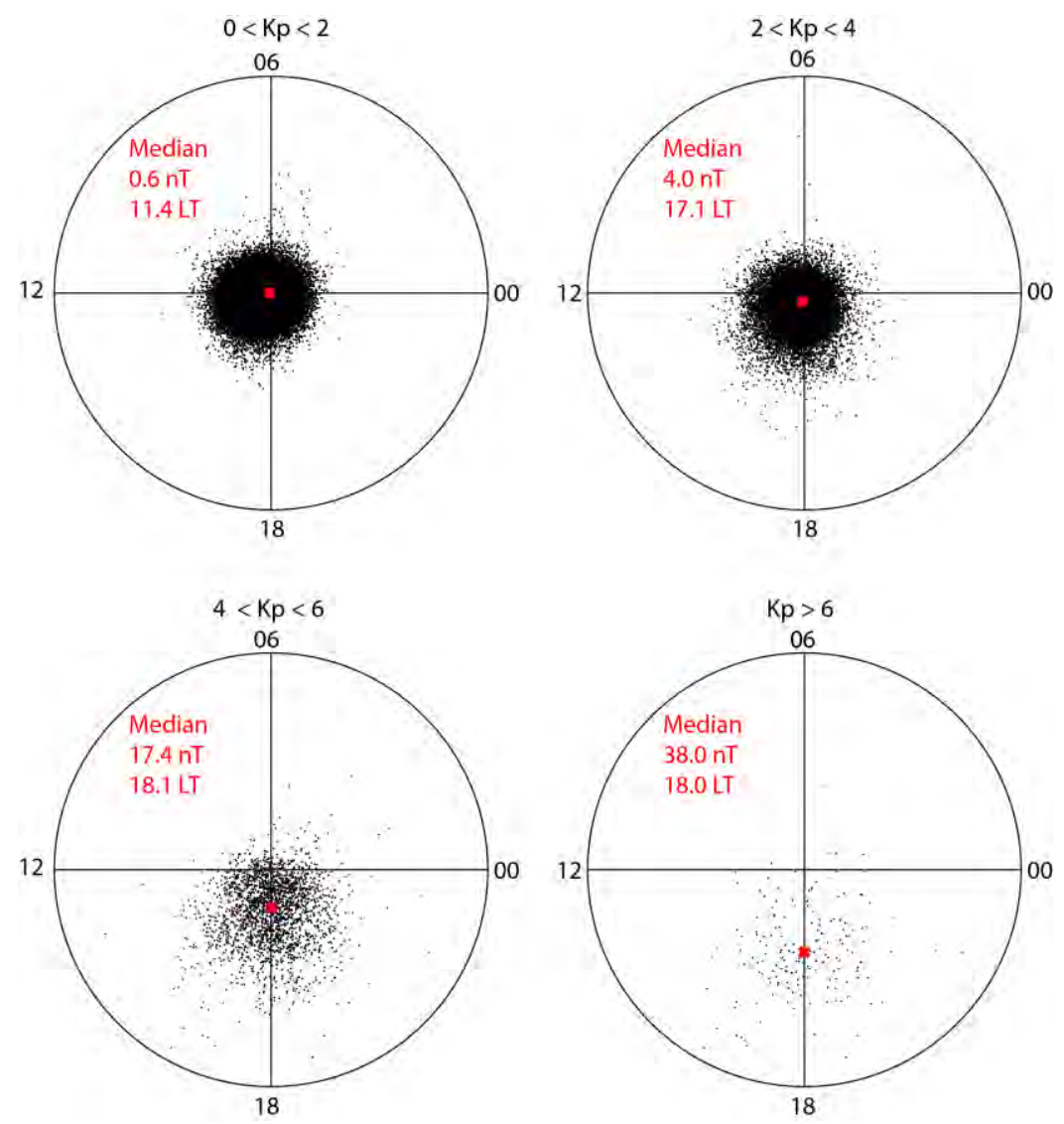

Fig. 6 Dependence of ring current asymmetry on magnetic activity. The centres of fitted circles shift progressively towards the dusk sector with increasing activity.

The black dots scatter quite a bit, but that is mainly due, in particular for quiet periods, to a degrading calibration of the C/NOFS magnetometer after the end of the CHAMP mission, September 2010. From the median values listed in the dials we can see that well-centred circles result during quiet periods. There is clear evidence for a shift of the centre towards the evening sector at higher magnetic activity and the amount of displacement progressively increases with the disturbance level. Already at moderate activity $(K p \sim 5)$ the centre is shifted by more than $17 \mathrm{nT}$. Although no super storms $\left(\mathrm{D}_{\mathrm{ST}}<-300 \mathrm{nT}\right)$ occurred during the considered 4 years, large asymmetries between dawn and dusk deflections of more than $75 \mathrm{nT}$ are observed during active periods.

Similar results concerning the asymmetry of the ring current effect have been derived from ground-based observations. Newell and Gjerloev (2012) made use of a large number of 
magnetometers from the SuperMAG data repository. A total of 98 geomagnetic stations are used to derive the SuperMAG ring current index, SMR. It is a quantity comparable to $\mathrm{D}_{\mathrm{ST}}$ or SYM-H but provides local time resolution from four sectors (SMR-00, SMR-06, SMR-12, SMR-18). For studying the typical magnetic storm evolution all storms during the years 1997 2007 which exceeded $D_{\mathrm{ST}}=-80 \mathrm{nT}$ were considered. By means of a superposed epoch analysis the authors determined the average evolution of the SMR indices in the four local time sectors, using the start of main phase (decrease of field strength) as key time $(\mathrm{t}=0)$. Figure 7 shows the average curves of storm-time signals at low latitudes for the four indices centred at the magnetic local time (MLT) sectors 00 MLT, 06 MLT, 12 MLT, and 18 MLT. All four indices exhibit a southward deflection after onset but with different slopes. About 10 hours into the storm minima are reached. Largest field depressions are found within the 18 MLT sector and smallest around 06 MLT. The difference in peak amplitude between dawn and dusk amounts to about $30 \mathrm{nT}$. For the noon and midnight sectors comparable excursions are observed. During the recovery phase the four curves converge again.

All these results are fully compatible with the C/NOFS observations reported by Le et al. (2011). The difference in peak deflection between SMR-06 and SMR-18 of $30 \mathrm{nT}$ corresponds to a shift of circle centre by $15 \mathrm{nT}$. When looking at Figure 6 we see that C/NOFS finds significantly larger shifts for high magnetic activity. This is probably due to the individual interpretation of every orbit as compared to the averaging of time series from many storms. The duration of storms can vary largely from case to case, and the applied averaging heavily reduces peak values. We thus may conclude that the typical storm-time asymmetry is clearly larger than deduced from the averaged SMR evolution presented here. The comparison between satellite and ground-based observations allows for another conclusion. Since the data from above and below the ionosphere show the same asymmetry of ring current effect, ionospheric currents cannot be the cause for the dusk sector intensifications during storms. Responsible currents have to flow above the C/NOFS orbit $(>850 \mathrm{~km})$. 


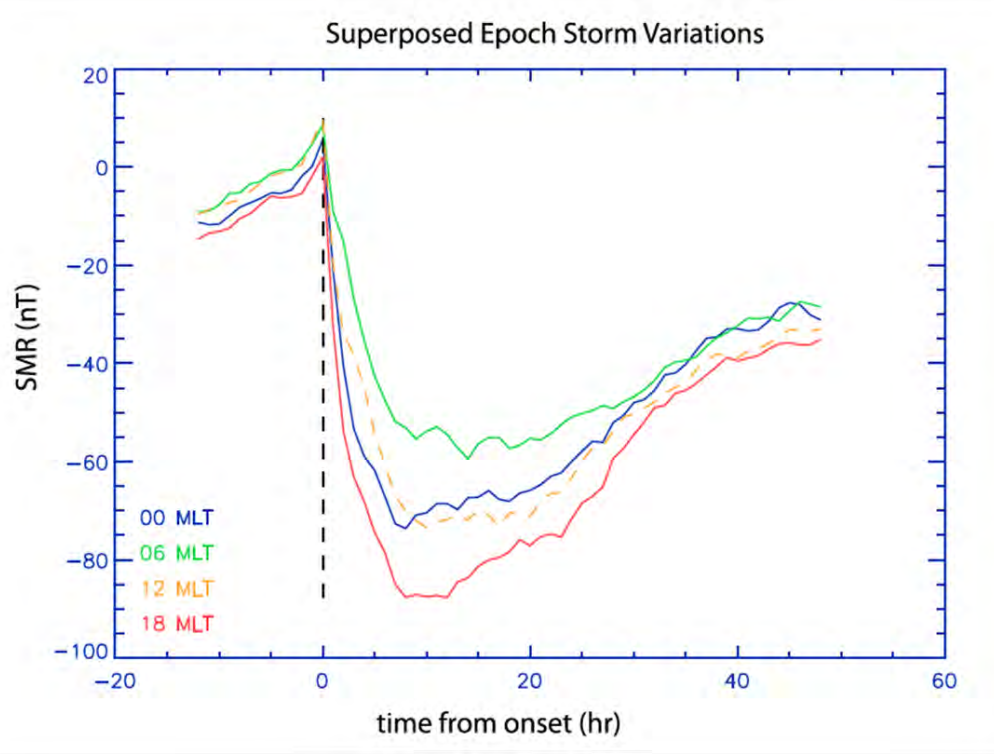

Fig. 7 Average evolution of the storm-time magnetic signal at middle latitudes separately for four different magnetic local time sectors. A superposed epoch analysis has been applied, using the start of main phase as key time $(\mathrm{t}=0)$. (after Fig. 7 from Newell and Gjerloev, 2012)

Near-Earth observations may suggest that during the storm main phase an additional partial ring current is forming in the evening sector. It would be desirable to prove this inference by direct measurements in the magnetosphere. New opportunities for in situ observation of the ring current arose with the advent of the Cluster constellation mission. This fleet of four satellites enables during perigee passes direct measurements of current density in the ring current area. Zhang et al. (2011) derived current density estimates for all local times. They considered Cluster observations from the periods 18 March to 14 June 2002 and 14 July 2003 to 27 April 2004. As can be seen in Figure 8, largest current densities are found in the sector 06 to 09 MLT, while small current densities are observed around 18 MLT. This finding seems to be in stark contrast to our expectations from magnetic effects observed at LEO and on ground. However, when interpreting the Cluster current density estimates one has to take into 
account that not the whole volume of the ring current region has been sampled by the Cluster constellation but just individual north-south passes are evaluated. The total current distribution may well be different from the derived local current density. Both, the radial current density profile, as well as the north-south extent of the current carrying volume may vary with local time. Further studies are needed focussing more on the total current intensity in the different sectors rather than the current density profiles along satellite tracks. Such results should be more significant for comparisons with ground-based magnetic field observations.

In a comprehensive study of the ring current Le et al. (2004) used data of the spacecraft ISEE, AMPTE/CCE and Polar for deriving statistical results on the longitudinal distribution of currents. As the main conclusion the authors claim that a significant fraction of the ring current is partial, flowing only within a limited longitudinal region and must be diverted out of the equatorial region as FACs to close in the ionosphere. During quiet times the azimuthal current strength is highest on the nightside and lowest on the dayside. With increasing activity the intense current moves towards the duskside. The ring current distribution deduced from their in situ magnetic field data indicate that the current intensity varies strongly through longitude sectors, and only $20 \%$ can be regarded as symmetric under moderate storm conditions. 


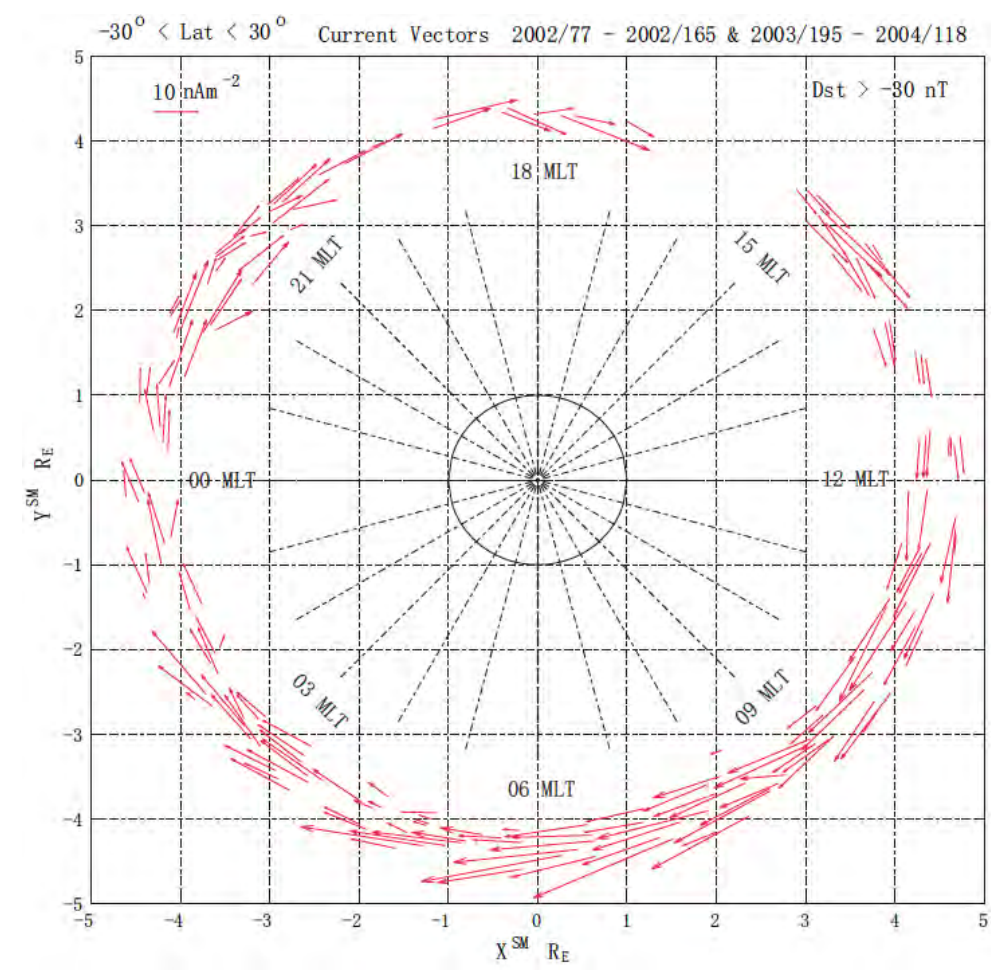

Fig. 8 Local time distribution of in situ Cluster ring current density measurements. (after Fig. 4 of Zhang et al., 2011)

Another technique of indirect ring current intensity estimation is counting the energetic neutral atoms originating from the ring current region. An instrument that can provide this information, the Energetic Neutral Analyser (ENA), has been flown on the IMAGE satellite. Some energetic ions (mainly Hydrogen or Oxygen) suffer charge exchanges with neutral Hydrogen atoms in the magnetosphere. After the colliding ion has gained an electron from the neutral, it can fly over large distances along a straight line, at a velocity according to its initial energy, because it is unaffected by the ambient magnetic and electric fields, and collisions are very rare in the outer magnetosphere. From the direction of arrival one can deduce the source region of the particle. The flux of emitted neutral particles is proportional to the density of ions in the source region. Since the energy is largely conserved through the process of charge exchange, also the energy spectrum of particles within the source region can be recovered. 
According to the well-known Dessler-Parker-Sckopke relation the total current is proportional to the total energy stored in the ring current (Dessler and Parker, 1959; Sckopke, 1966). ENA instruments are able to obtain a complete picture of the energetic particle distribution in the ring current at all longitudes and radial distances. Initial results of the ENA instrument on board IMAGE have been published by C:son Brandt et al. (2002). Figure 9 shows one example of ENA measurements during a magnetic storm on 4 October 2000. At the time of energetic neutrals recording, 18:00 UT, the activity had reached $\mathrm{D}_{\mathrm{ST}}=-140 \mathrm{nT}$. In the energy range around $30 \mathrm{keV}$, where most of the ring current particles can be found, highest fluxes come from the local time sector 00 to $06 \mathrm{MLT}$ and a radial distance between 3 and $5 \mathrm{R}_{\mathrm{E}}$. A rather similar distribution can be found for the higher energetic particle. The authors have investigated 17 more events in the time frame 2000-2002 and found peak ENA counts in the post-midnight sector practically in all cases. The higher density of energetic ions in the postmidnight/morning sector suggest a stronger ring current in that region, which is similar to the in situ current measurements of Cluster. However, near-Earth observations suggest a somewhat different local time distribution of the current intensity. This apparent incompatibility may be explained by additional currents like field-aligned and/or magnetopause currents that cause the enhanced magnetic deflections in the dusk sector during storm main phases (see Haaland et al., 201 ). More coordinated space - ground studies are needed for obtaining a more realistic picture of the magnetospheric current geometry during storms. Although only quiet periods are considered for main field modelling, the actual "partial ring current" configuration can have on average a non-negligible influence on the results that may vary with season and/or solar cycle if not considered properly. 


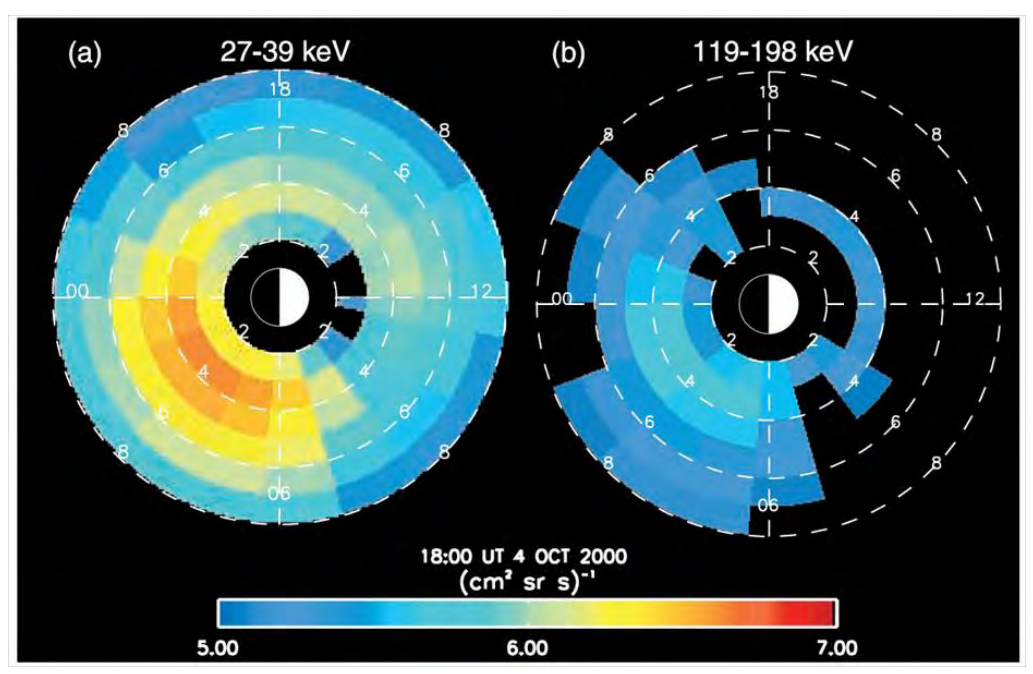

Fig. 9 Energetic Neutral Atom image of the ring current region during a storm on 4 October 2000. Highest fluxes of energetic particles emerge from the sector 00 to 06 local time (after Fig.1 of C:son Brandt et al., 2002).

\subsection{Résumé of ring current magnetic field effect}

The storm time index $\mathrm{D}_{\mathrm{ST}}$ is widely used to characterise the strength of a magnetic storm. Commonly its value is related to the intensity of the magnetospheric ring current. In spite of its usefulness in general we have outlined some of the weaknesses of this index, in particular when it comes to quantifying the ring current effect during low activity periods, which are of interest for magnetic field modelling efforts. A major problem seems to be the reliable determination of quiet-time base lines for the magnetic observatories contributing to $\mathrm{D}_{\mathrm{ST}}$. A certain amount of ring current is always flowing even during quiet times. This basic level of field contribution is difficult to derive from ground-based observations, but can be determined from satellite recordings on polar orbits. We have found that the $\mathrm{D}_{\mathrm{ST}}$ values commonly underestimate the quiet-time ring current effect. Around solar maximum years the deficit amounts on average to about $10 \mathrm{nT}$, which may already be of interest for space science studies. The difference almost disappears at very low solar activity. In detail, however, the 
differences are quite variable. Here we have presented an alternative, the RC index, based on a more sophisticate approach for deriving a reliable representation the magnetospheric ring current effect.

A topic, relevant at least during magnetic storms, is the longitudinal asymmetry of the ring current intensity. It is known since long that the magnetic field depression during a storm is strongest in the local time sector around 18 LT. This has commonly been associated with the formation of a partial ring current that enhances the total current in a certain sector. However, recent in situ current density measurements by Cluster sense weak currents in the evening sector and peak current densities around early morning. A similar result is inferred from energetic neutral atom measurements that confirm highest fluxes of energetic ions in the postmidnight/early morning sector. In contrast, Le et al. (2004) deduce from field measurements in the magnetosphere a ring current distribution more similar to the ground-based results. More dedicated studies are needed for reconciling the apparent incompatibilities between ground-based and in situ measurements.

\section{Characteristics of magnetospheric tail current}

On the nightside the magnetosphere is pulled out by the solar wind into a long tail, extending several $100 \mathrm{R}_{\mathrm{E}}$ into space. Particular current systems are responsible for the shape of the magnetospheric tail (see Fig. 1). At a certain distance down-tail of about $10 R_{E}$ the magnetosphere has the shape of a tube, opening up with a flaring angle of typically $5^{\circ}$ at 30 $\mathrm{R}_{\mathrm{E}}$. The main tail current configuration is schematically shown in Figure 10. The cross-tail current (also called neutral sheet current) is flowing at about the centre of the tail from the morning to the evening side. At the dusk side magnetopause the current is diverting, flowing over the northern and southern lobes back to the dawn side. These two current loops generate 
rather homogeneous magnetic fields, which point towards the Earth in the northern lobe and away from the Earth in the southern lobe. At the position of Earth the resulting magnetic field points southward, perpendicular to the cross-tail plane. In Figure 10 red arrows indicate magnetic field directions. The sizes of the tail and Earth on that figure are approximately to scale. The small size of Earth compared to the tail dimensions implies that no significant differences can be expected between magnetic effects on the day and nightside. We thus may assume a homogenous field distribution caused by the tail currents.

The orientation of the tail axis is closely controlled by the direction of the solar wind.

Therefore it is on average aligned with the Sun-Earth line plus a small aberration angle of $4.3^{\circ}$ caused by the orbital speed around the sun. About this line the tail can easily be rotated. For that reason it follows the tilt of the geomagnetic dipole axis in the plane perpendicular to the Sun-Earth line. As a consequence of that behaviour near-Earth magnetic effects of magnetotail currents can efficiently be described in GSM coordinates. They are primarily confined to the -z component. There is some additional effect of the IMF By component that is twisting the tail about its axis (e.g. Cowley, 1981; Tsyganenko and Fairfield, 2004). This causes also magnetic field deflections at Earth in the y component.

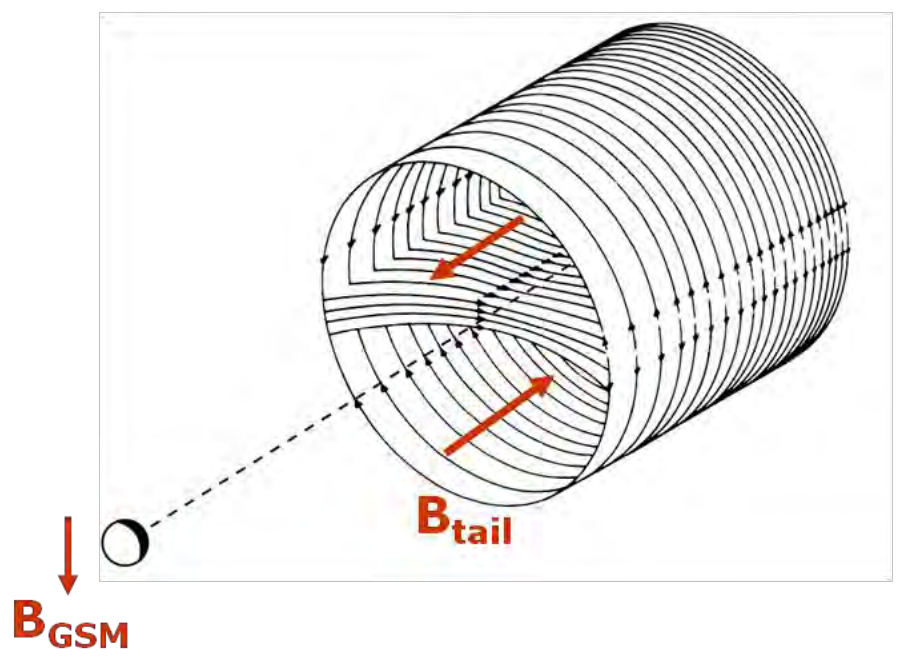


Fig. 10 Schematic drawing of the magneto-tail current configuration. The Earth is drawn at a distance of $15 \mathrm{R}_{\mathrm{E}}$. Red arrows represent the generated magnetic field directions. (modified after Olsen, 1982)

\subsection{Magnetic effect of the magneto-tail}

In the past there have been attempts to estimate the near-Earth magnetic effect of magneto-tail currents as part of geomagnetic field modelling efforts. Maus and Lühr (2005) were the first to derive from Ørsted and CHAMP data magnetic field contributions in GSM coordinates which were related to magneto-tail currents. They report for the maximum years of solar cycle 23 an average stable value of $-12.9 \mathrm{nT}$ well aligned with the GSM z component. In addition they confirmed a weak dependence of GSM y on the IMF By component $(y=0.23 B y)$. Such a leakage of the IMF into near-Earth space has earlier been noted by Lesur et al. (2005) and is due to the twisting of the magneto-tail (e.g. Tsyganenko and Fairfield, 2004). Nowadays it is common practice in geomagnetic field modelling to separate the external field contributions into their SM and GSM parts (e.g. Olsen et al., 2005, 2014; Lühr and Maus, 2010; Alken et al., 2015). However, there is still some uncertainty about the amplitude of the tail current effect. Quiet-time values ranging from 8 to $13 \mathrm{nT}$ are quoted in the different studies. A more reliable determination of that quantity would be desirable.

The Earth performs periodic motions (rotation and seasonal tilt of spin axis) relative to the stable GSM field from the magneto-tail currents. At a fixed point on Earth surface the tail field causes diurnal and annual variations. Maus and Lühr (2005) had already compared the expected annual variations of about $\pm 5 \mathrm{nT}$ caused by the tail currents with the annual baseline variations recorded at five observatories. They found a good agreement in all cases. Here we try to give a more general picture of the apparent field variations. 

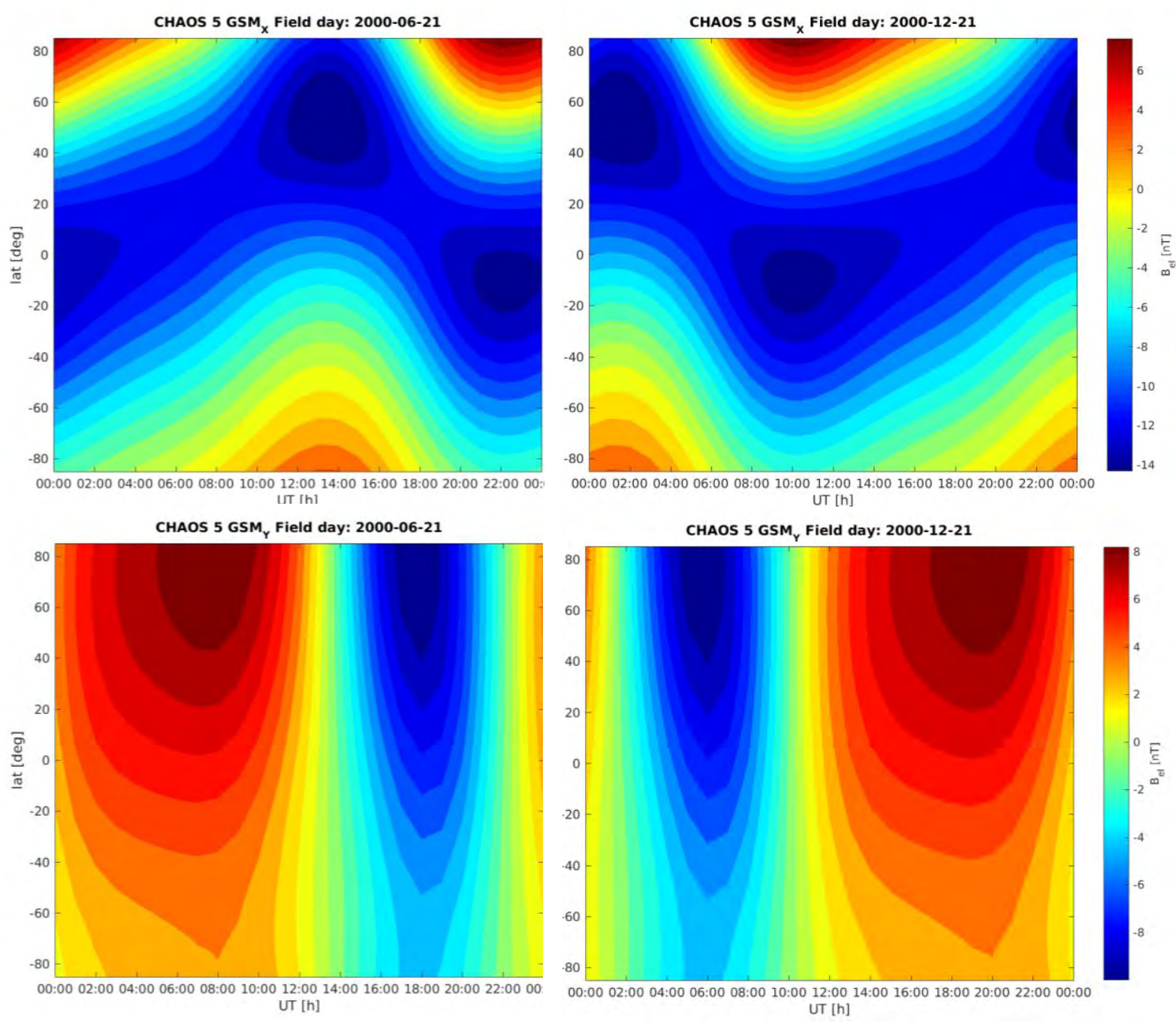

Fig. 11 Diurnal variation caused by the magnetic effect of the magneto-tail currents. The left column shows for the different latitudes the deflections of horizontal components at June solstice and the right column at December solstice. The presented results are valid for the Greenwich meridian.

Figure 11 shows the global distribution of expected diurnal variation caused by tail currents according to the CHAOS-5 geomagnetic field model (Finlay et al, 2015). In that model only the dominant external dipole term in GSM coordinates is of importance. Plotted are the deflections of the northward (top) and eastward (bottom) components. June and December solstice days have been chosen because largest diurnal variations occur during these days. We have selected, as example, a profile along the Greenwich meridian. Here the UT and LT times 
are identical. At other longitudes the latitude dependence is somewhat different. Quite large variations appear at northern mid latitudes. In the eastward component the diurnal deflection amounts to $\pm 8 \mathrm{nT}$, and it is somewhat smaller in the northward component. It is interesting to note that the presented variations of both components at northern mid latitudes are in phase with the typical Sq variations during June solstice, but in anti-phase around December solstice (e.g. Yamazaki et al., 2016, this issue). That means, the effect of the magneto-tail currents reaches $10 \%$ to $20 \%$ of the Sq amplitude during solstice seasons. When estimating Sq-related ionospheric currents from magnetic observatory data the influence of tail currents should first be removed.
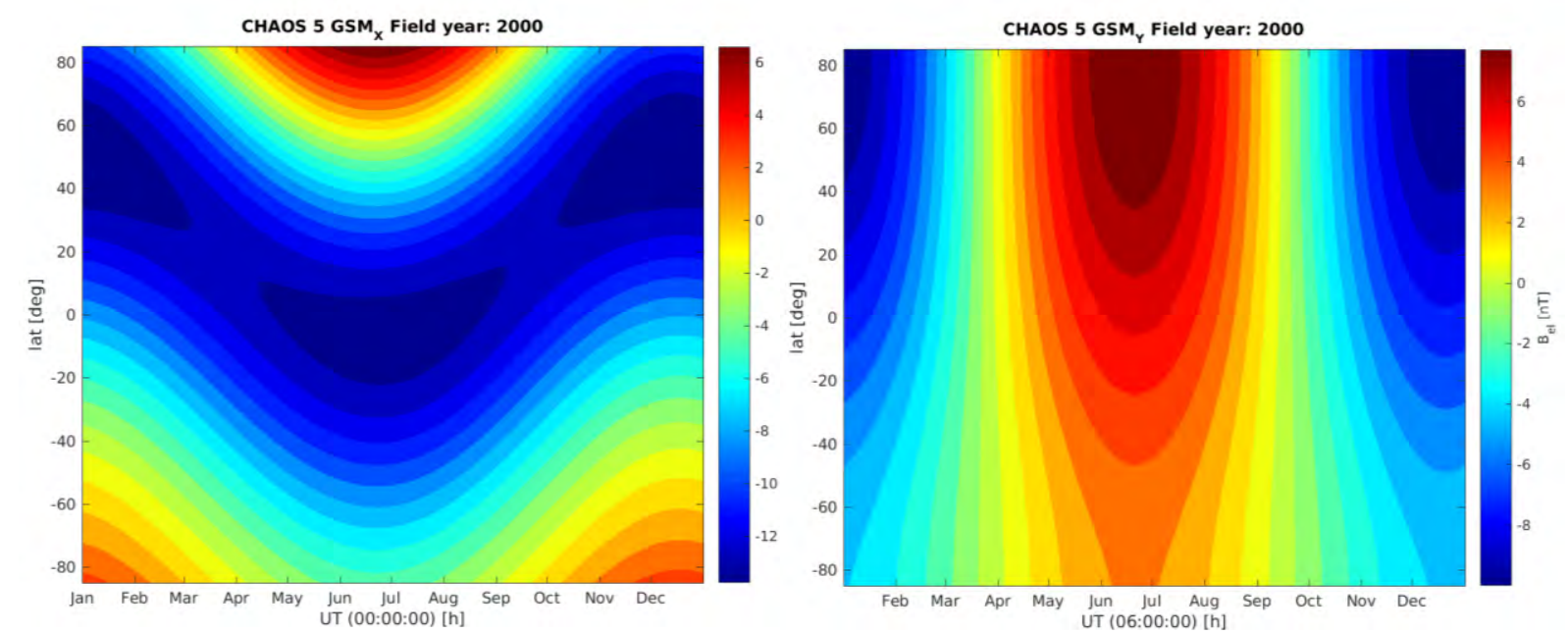

Fig. 12 Annual variation caused by the magnetic effect of the magneto-tail currents. The left frame shows for the different latitudes the deflections of the northward components at midnight and the right frame for the eastward component at 06 local time. The presented results are valid for the Greenwich meridian.

There is also an annual variation caused by the magneto-tail currents. For quiet night times we can find, according to the CHAOS-5 model, variations in the northward component over the course of a year of up to $10 \mathrm{nT}$ peak-to-peak at mid latitudes. In the eastward component 
largest annual variations appear during the morning hours. Therefore the distribution at 06 local time has been presented in the right frame of Figure 12. In the northern hemisphere deflections are exceeding $\pm 6 \mathrm{nT}$. These so-called annual variations of the baselines are known for quite some time (e.g. Campbell, 1984) but could not be explained correctly in those years.

\subsection{Parameterisation of the magneto-tail current effect}

For a proper isolation of the magneto-tail current effect in geomagnetic field modelling approaches it would be desirable to have a quantity that can be used as a proxy for the current strength. Since such an index is not available, we may try an indirect method for quantifying the tail current effect. In the Earth's magnetosphere, at altitudes above about $1000 \mathrm{~km}$, collisions between particles occur rather seldom. Under these conditions the electric conductivity may become very large, and a good approximation for the dynamics of such a plasma is offered by the frozen-flux theorem. This means, the amount of magnetic flux does not change along field lines. We can make use of this characteristic for quantifying the magnetic field strength in the magneto-tail. At high latitudes the auroral oval forms. Closed magnetic field lines reaching into the magnetosphere thread this region. The region poleward of the auroral oval is called the polar cap. Magnetic field lines origination from the polar cap are regarded 'open'. They are connected on one side to the Earth and on the other end to the solar wind. All field lines from the northern polar cap enter the northern magneto-tail lobe and those from the southern polar cap lead through the southern lobe. According to the frozen-flux theorem the magnetic flux integrated over the area of each of the two polar caps should be equal to the magnetic flux threading the corresponding lobes of the tail. In case of a spherical polar cap the magnetic flux, $\Phi_{P C}$, can be calculated as

$$
\Phi_{P C}=\pi\left(R_{E} \sin \theta_{P C}\right)^{2} B_{P C}
$$


where $\theta_{P C}$ is the magnetic co-latitude of the polar cap boundary and $B_{P C}$ is the mean magnetic field strength within the polar cap. Equivalently, the magnetic flux within a tail lobe, $\Phi_{\text {tail }}$, can be expressed as

$$
\Phi_{\text {tail }}=1 / 2 \pi R_{\text {tail }}^{2} B_{\text {tail }}
$$

where $R_{\text {tail }}$ is the radius of the magneto-tail with a typical value of $20 \mathrm{R}_{\mathrm{E}}$ and $B_{\text {tail }}$ is the mean field strength within the tail lobes. By equating (7) and (8) we can solve for the field strength in the tail lobes.

$$
B_{\text {tail }}=2 \Phi_{P C} /\left(\pi R_{\text {tail }}^{2}\right)
$$

If we know the polar cap boundary, we can estimate the field in the magneto-tail and with that the expected magnetic effect at Earth. A typical value for the colatitude is $\theta_{P C}=15^{\circ}$ and the mean field strength is around 55,000 nT. With these numbers we obtain an open magnetic flux during normal days of $\Phi_{P C}=486 \cdot 10^{6}$ Vs. With the help of Eq. (9) we can get a value for the magnetic field in the tail lobes, $B_{\text {tail }}=19 \mathrm{nT}$. In situ observations, e.g. from Cluster, confirm that $20 \mathrm{nT}$ is a typical field strength observed in the tail lobes. For more details of the polar cap to magneto-tail relation see Hughes (1995).

The simple geometry magneto-tail currents, as shown in Figure 10, allows to estimate the magnetic effect at Earth. For a circularly shaped magnetopause and a cross-tail current in the middle, which splits up evenly on the dusk side into return currents over the northern and southern lobes, an analytic expression can be given. This current configuration is assumed to start at a distance of $10 R_{E}$ from Earth and extends into infinity (any part beyond $100 R_{E}$ has no significant impact). For this symmetric configuration we get at Earth only a contribution along the GSM z component, which can be calculated as

$$
B_{z}=-\frac{\mu_{0} J}{2 \pi} \ln \frac{R_{\text {tail }}+\sqrt{r_{0}^{2}+R_{\text {tail }}{ }^{2}}}{r_{0}}
$$


where $J$ is the current density of the neutral sheet cross-tail current and $r_{0}=10 \mathrm{R}_{\mathrm{E}}$ is the distance to Earth. When considering a tail field of $20 \mathrm{nT}$ we can estimate the sheet current density within the cross-tail neutral sheet $\left(2 B_{\text {tail }}=\mu_{0} J\right.$, effects of the currents in the two lobes largely compensate). With the resulting $J=32 \mathrm{~mA} / \mathrm{m}$ we get a magnetic effect of $B z=-9.2 \mathrm{nT}$ at Earth.

It is known that the size of the magnetospheric tail and the intensity of the cross-tail current increases with magnetic activity. During times of southward IMF magnetic flux is opened on the dayside and added to the tail. It would be desirable to have a parameter that can be used to track the change of magnetic flux in the tail. Here we propose to use the magnetic flux of the polar cap for this purpose. Recently there has been an empirical model of the auroral oval, termed CH-Aurora-2014, introduced by Xiong and Lühr (2014), in which the poleward and equatorward boundaries of the oval are derived from the latitude distribution of small-scale field-aligned currents. The intensity profile of small-scale FACs is deduced from CHAMP or Swarm satellite magnetic field observations. With the help of a correlation analysis we identified the solar wind to magnetosphere coupling function defined by Newell et al. (2007), subsequently termed merging electric field, $E_{m}$, as the most suitable quantity controlling the position of auroral oval boundaries. The field line merging efficiency at the dayside magnetopause closely controls this coupling function. More details on the determination of the boundaries can be found in Xiong et al. (2014). Figure 13 shows examples of average auroral oval distributions for three magnetic activity levels. 

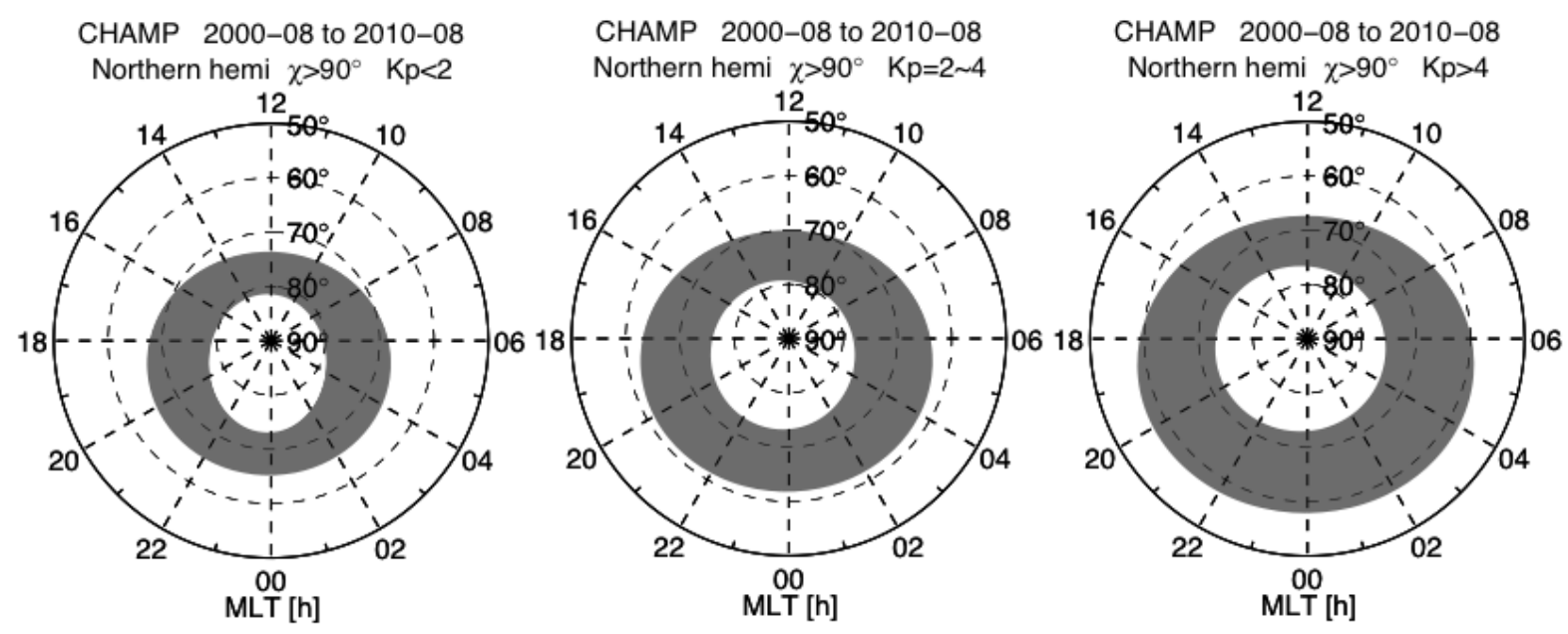

Fig. 13 Magnetic latitude and local time distribution of the auroral oval for three different magnetic activity levels (reproduced from Fig. 7 of Xiong et al. (2014)).

In order to be able to make predictions of the tail field effect at Earth we first have to calibrate the functional relation between estimated polar cap magnetic flux and the observed magnetic field in GSM coordinates. For this purpose we used observations from the CHAMP mission during the years from 2000 to 2010. Data over at least one year are needed for separating sufficiently well the closely aligned contributions of the ring current (in SM) from that of the tail current (in GSM). The CHAMP dataset has been divided into six activity classes determined by periods of prevailing merging electric fields $(E m)$ centred at $(0.5,1.5,2.7,4.2$, 6.0, $9.7 \mathrm{mV} / \mathrm{m})$. For obtaining the external field contributions we first subtracted the CHAOS4 core and crustal field model (Olsen et al., 2014) from the CHAMP magnetic field data. Then the residuals of the six activity classes were interpreted separately. To each class of residuals we applied a spherical harmonic analysis, where the expected ring current activity in SM frame was parameterised by the RC index, as described in section 3.1. For improving the fit between the CHAMP-derived SM values and the ground observations we allowed for a scaling factor applied to the RC index and an additive bias term constant for all six activity 
classes. Of particular interest here are the derived external contributions in GSM frame. For each of the classes we obtained a value well aligned with the GSM $-\mathrm{z}$ direction.

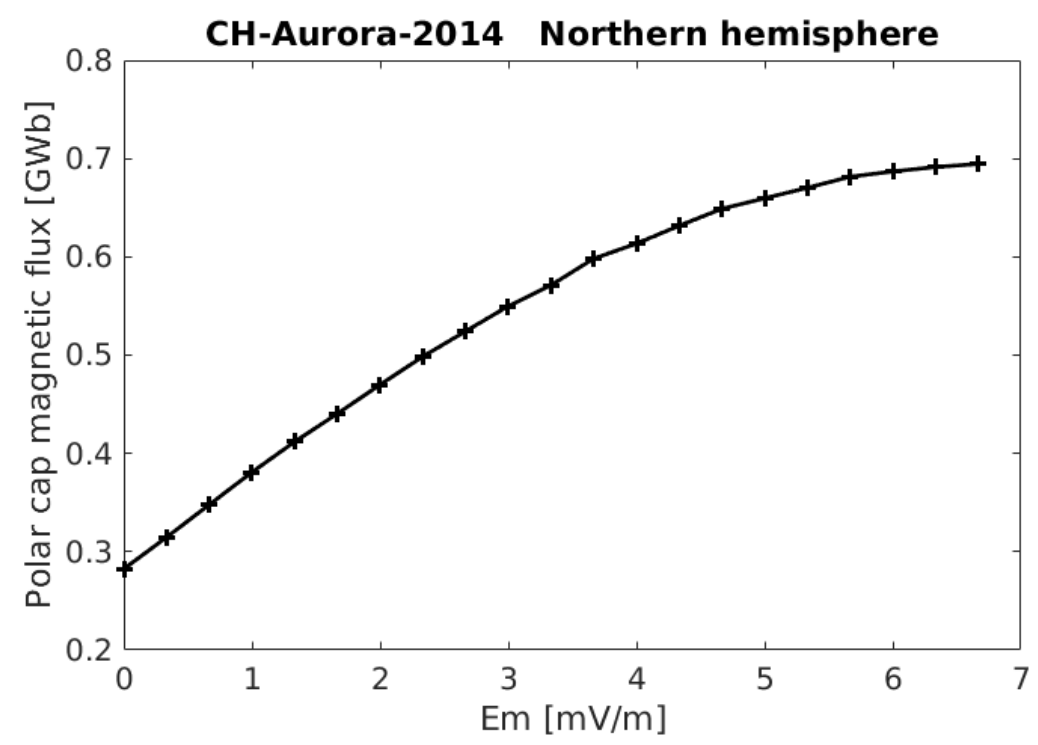

Fig. 14 Increase of the polar cap magnetic flux with growing merging electric field, Em.

The other quantity of interest is the magnetic flux confined to the polar cap. With the help of the CH-Auroral-2014 model (Xiong and Lühr, 2014) we can compute the open magnetic flux for any merging electric field value. Figure 14 shows the increase in polar cap magnetic flux with growing $E m$ values. As can be seen in the figure, the magnetic flux starts to saturate for $E m$ values larger than $4 \mathrm{mV} / \mathrm{m}$. There are obviously active processes that slow down field line merging and adding of more magnetic flux to the magneto-tail when the merging electric field exceeds a certain value.

Of particular interest for this study is the relation between open magnetic flux and magnetic field effect from the tail currents. Results obtained from our six activity classes are shown in 
Figure 15. As can be seen there exists an excellent linear relationship between these two quantities. This result is very convincing because both quantities have been derived fully independently. From Figure 14 we know that the magnetic flux goes into saturation for large merging electric fields. The same behaviour is obviously true for the neutral sheet current in the tail. In any case, the strict linear relation confirms the theoretically inferred connection between polar cap size and open flux in the magnetospheric tail.

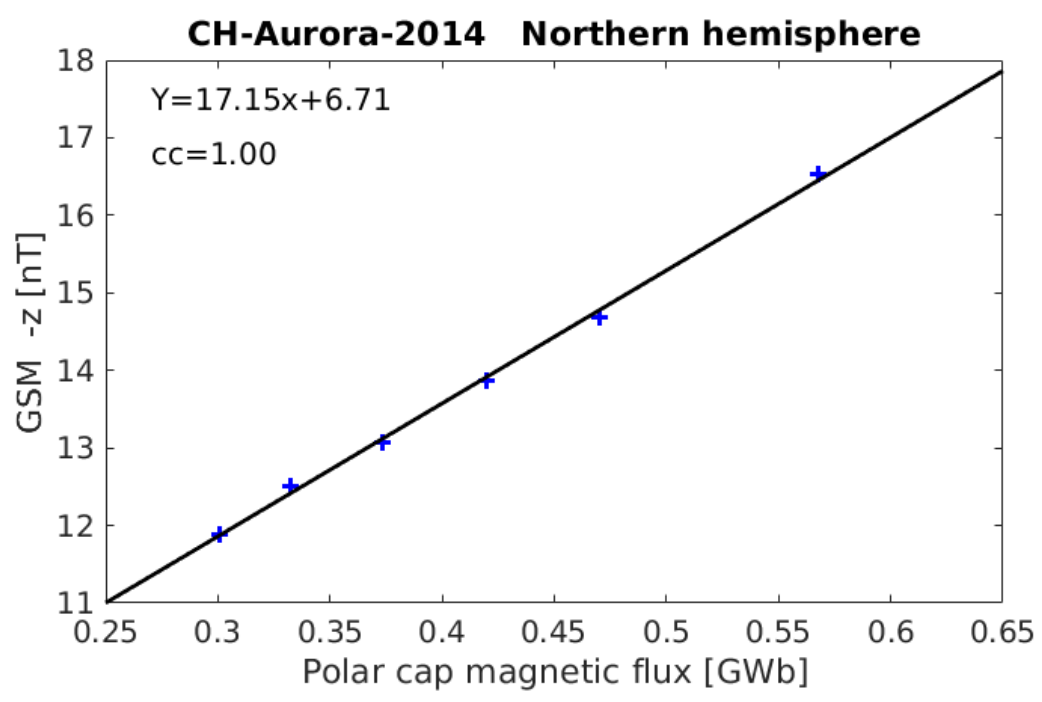

Fig. 15 Ratio between polar cap magnetic flux and the magnetic effect of tail currents at Earth observed in the GSM - Bz component. The linear dependence confirms the theoretically expected relation between the two quantities.

The regression function, listed in the top of Figure 15, provides us in principle with a formula for estimating the magnetic contribution from the magneto-tail. Somewhat surprising is the rather large bias value of $6.7 \mathrm{nT}$ in the equation. This would mean, even if the polar cap size approaches zero, there is still an appreciable magnetic disturbance from the magneto-tail, which makes no sense. There are a number of reasons that may cause this artefact. For low 
magnetic activity our estimate of the magneto-tail effect is about $12 \mathrm{nT}$. There have been earlier publications quoting 8 to $9 \mathrm{nT}$ for the quiet-time tail effect (e.g. Olsen et al., 2005; Lühr and Maus, 2010). Also our first-order estimate of the magneto-tail current effect, presented above, gives $9 \mathrm{nT}$ at Earth. This would bring down the bias to below $3.5 \mathrm{nT}$. It is notoriously difficult to distinguish properly with the spherical harmonic analysis between the quiet-time contributions from the ring current and the tail currents. Therefore an interchange of a few nT between the two frames can easily occur. Luckily, such an exchange between the SM and GSM bias values has no significant effect on the quality of the geomagnetic field models. Another contribution to the field bias could result from the estimated amount of open flux. From a comparison of the $\mathrm{CH}$-Arora-2014 model with ultraviolet images taken by the IMAGE satellite Xiong and Lühr (2014) deduced that the CHAMP model underestimates the diameter of the polar cap on average by $0.5^{\circ}$. Taking this into account reduces the apparent bias in the relation by another $1.5 \mathrm{nT}$. Because of the excellent linear relation between the two independently estimated quantities, open flux and magneto-tail field effect, we regard the obtained linear slope as reliable. Considering all these arguments we suggest to use the $\mathrm{CH}$ Aurora-2014 model for estimating the magnetic flux, $\Phi$, within the northern polar cap and predict the magneto-tail effect at Earth in GSM coordinates by the function

$$
B z=-(17.15 \Phi[G W b]+3.5[n T])
$$

The resulting magnetic fields (in nT) can be used in geomagnetic field modelling approaches for parameterising the contributions from the magnetospheric tail currents. 


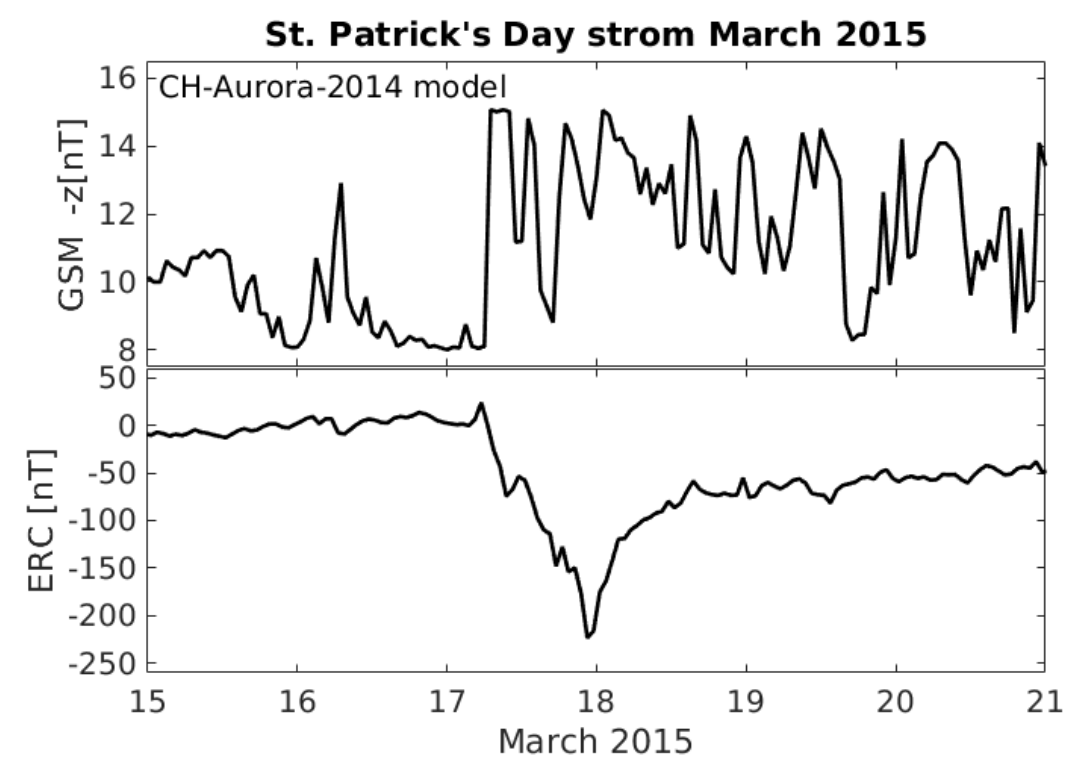

Fig. 16 Examples for the magneto-tail current and ring current effects at Earth. Here the days around the St. Patrick's Day storm have been chosen.

Figure 16 shows an example for the magnetic field contributions from tail and ring currents. It is quite evident how different the characters of these two contributions are. The effect of the ring current is much larger. Changes take place on longer time scales. At quiet times this effect reduces to small values. Due to its large dynamics the ring current effect has to be considered carefully when separating the different field contributions. In contrast, the magnetospheric tail currents are recovering generally much faster, on the order of few hours, to quiet-time configuration, but there occur also sudden increases. The St. Patrick's Day storm is somewhat special in that respect since the magnetic activity remained elevated for several days after the main phase. Another feature of the magneto-tail effect, the range of variations seem to stay within $5 \mathrm{nT}$ while the basic level of magnetic field at Earth is about twice as large. For a proper consideration of the contribution from the magneto-tail it is advisable to take into account the estimated field strength in the GSM z component in geomagnetic modelling efforts. 


\subsection{Résumé of magneto-tail current field effects}

The magnetospheric tail is an important electrodynamic region of near-Earth space. Its shape is formed by the balance between solar wind kinetic pressure on the outside and the magnetic pressure on the inside. For that reason its orientation is well aligned with the solar wind flow. During times of southward IMF new magnetic flux is opened on the dayside magnetopause and added to the tail. Eventually the flux piled-up in the tail reconnects and offloads energy and momentum within the substorm process. All the currents accompanying these processes and shape reconfigurations generate magnetic fields observable at Earth. For high-resolution geomagnetic field modelling a proper consideration of the field contributions from the tail is essential. In recent years it has been found that magnetic fields from the tail, of order $10 \mathrm{nT}$, are well organized in GSM coordinates as compared to the ring current effects in SM frame.

Unfortunately there exists no index that quantifies the intensity of tail currents. Here we introduce a possible proxy for that purpose. The amount of open flux in the polar cap is assumed to be equal to the magnetic flux in a tail lobe. Based on field-aligned current distributions a model of the auroral oval boundaries has been developed from CHAMP and Swarm observations. This model (CH-Aurora-2014) allows to predict the actual position of the polar cap boundary. With the help of that the open flux in the two hemispheres can be calculated. For checking the validity of the inferred relation between tail magnetic flux and near-Earth magnetic effect we performed a statistical analysis over many years comparing the two quantities for different levels of activity. The excellent linear relation resulting from our calibration confirms that the estimated polar cap open flux can be used to represent the 
temporal evolution of the magneto-tail current effect. Future applications may demonstrate the suitability of this proxy for geomagnetic field modelling.

\section{Summary and outlook}

In this article we reviewed features of large-scale magnetospheric currents. We have not focused on the details of physical processes responsible for their existence. Rather we try to interpret the magnetic signatures they cause near-Earth. Dedicated magnetic field survey missions like Ørsted, CHAMP and now Swarm have provided deeper insight into the various contributions to the geomagnetic field. Conversely, higher demands arise from these accurate data for a proper separation between the source terms.

Important contributions to the geomagnetic field come from the magnetospheric ring current. It weakens the main field during times of enhanced magnetic activity. Traditionally the intensity of the ring current is represented by the storm-time, $\mathrm{D}_{\mathrm{ST}}$ index. However, our analysis revealed that the $\mathrm{D}_{\mathrm{ST}}$ index shows some deficits, in particular when it comes to characterise the ring current effect during quiet times. As an alternative we propose to use another index, the $\mathrm{RC}$, for quantifying the magnetic effect of the ring current. Direct comparisons with ring current estimates from CHAMP show an excellent agreement.

Another feature we investigated is the partial ring current. Prominent enhancements appear in the evening sector during active periods. We could confirm from ground and space-based observations the asymmetry of ring current effects between dawn and dusk sides. However, in situ measurements of the ring current density do not confirm the expected local time distribution of current intensity. This open issue needs further investigation in future.

The magnetic effect caused by magnetospheric tail currents is another topic we addressed. Good progress has been achieved since considering the tail current magnetic effect in GSM 
coordinates. Such a field causes at Earth surface various signatures (diurnal and annual variations), which were previously not understood.

So far there is no suitable index available for quantifying the intensity of magneto-tail currents. Here we propose to use the amount of open magnetic flux emanating from the polar caps as a proxy for that. Based on an empirical model of auroral oval boundaries we provide estimates of the magnetic flux in the tail lobes. A direct comparison of polar cap magnetic flux with the near-Earth magnetic effect of the tail currents confirms a linear relation between these two quantities. In future the polar cap magnetic flux may be used for parameterising the magneto-tail current contribution to the near-Earth magnetic field.

In our view a major issues to be address in future is the unsolved problem of the partial ring current. The traditional picture of enhanced ring current density within the dusk sector during magnetic storms may need revision. Here joint data interpretations from satellites in the magnetosphere and in low-Earth orbit, like Cluster and Swarm, may help to reconcile the contradicting results. In case of the magneto-tail currents more effort is warrant for refining the magnetic footprint on Earth. In particular seasonal effects due to tail deformation need to be investigated in more details. Also here the expertise of magnetospheric physics and geomagnetic field modelling has to be combined for achieving progress.

\section{Acknowledgements}

This article is based on results of the ISSI Workshop "Earth's Magnetic Field: Understanding sources from the Earth's interior and its environment". The authors thank the International Space Science Institute in Bern, Switzerland, its staff and directors for their support. 


\section{References}

Akasofu, S.-I. and Chapman, S.: On the asymmetric development of magnetic storm fields in low and middle latitudes, Planet. Space Sci., 12, 607-626, 1964.

Alken P, Maus S, Chulliat A, Manoj C (2015) NOAA/NGDC candidate models for the 12th Generation International Geomagnetic Reference Field. Earth Planets Space 2015 67:68

Campbell, W.H., 1984. An external current representation of the quiet nightside geomagnetic field level changes, J. Geomag. Geoelectr., 36, 257-265.

C:son Brandt, P., S. Ohtani, D. G. Mitchell, M.-C. Fok, E. C. Roelof, and R. Demajistre, Global ENA observations of the storm mainphase ring current: Implications for skewed electric fields in the inner magnetosphere, Geophys. Res. Lett., 29(20), 1954, doi:10.1029/2002GL015160, 2002.

Clauer, C. R. and McPherron, R. L.: Mapping of local time, universal time development of magnetosphere substorms using midlatitude magnetic observations, J. Geophys. Res., 79, 2812-2820, 1974.

Cowley, S. W. H.: Magnetospheric asymmetries associated with the y-component of the IMF, Planet. Space Sci., 29, 79-96, 1981.

de La Beaujardière, O., et al. (2004), C/NOFS: A mission to forecast scintillations, J. Atmos. Sol. Terr. Phys., 66, 1573-1591, doi:10.1016/j.jastp.2004.07.030.

Dessler and Parker, Hydromagnetic theory of geomagnetic storms, J. Geophys. Res., 64, 2239-2252, 1959.

Finlay, C. C., N. Olsen, and L. Toeffner-Claussen, DTU candidate field models for IGRF-12 and the CHAOS-5 geomagnetic field model, Earth, Planets, Space, 67, 114, doi:10.1186/s40623-015-0274-3, 2015.

Hamilton, D.C., Gloekler, G., Ipavich, F.M., Stüdemann, W., Wilken, B., Kremser, G., Ring current development during the great geomagnetic storm of February 1986, J. Geophys. Res., 93, 14 343-14 355, 1988.

Hughes, W. J.: The magnetopause, magnetotail, and magnetic reconnection, in: Introduction to Space Physics, edited by: Kivelson, M.G. and Russell, C.T., pp. 227-287, Cambridge Univ. Press, Cambridge, UK, 1995.

Iyemori, T.: Storm-time magnetospheric currents inferred from mid-latitude geomagnetic field variation, J. Geomag. Geoelectr., 42, 1249-1265, 1990.

Kivelson, M.G. and Russell, C., (eds.) (1995), Introduction to Space Physics, Cambridge Univ. Press, Cambridge, UK.

Langel, R.A., Estes, R.H., Mead, G.D., Fabiano, E.B., and Lancaster, E.R. (1980), Initial geomagnetic field model from MAGSAT vector data, Geophys. Res. Lett., 7:793-796. 
Langel, R.A. and Estes, R.H., Large-scale, near-earth magnetic fields from external sources and the corresponding induced internal field, J. Geophys. Res, 90, 2497-2494, 1985.

Le, G., C. T. Russell, and K. Takahashi (2004), Morphology of the ring current derived from magnetic field observations, Ann. Geophys., 22,1267-1295, doi:10.5194/angeo-221267-2004.

Le, G., W. J. Burke, R. F. Pfaff, H. Freudenreich, S. Maus, and H. L.hr ( 2011), C/NOFS measurements of magnetic perturbations in the low-latitude ionosphere during magnetic storms, J. Geophys. Res., 116, A12230, doi:10.1029/2011JA017026.

Lesur, V., Macmillan, S. \& Thomson, A. (2005), A magnetic field model with daily variations of the magnetospheric field and its induced counterpart in 2001, Geophys. J. Int., 160, 79-88.

Lühr, H and S. Maus (2010), Solar cycle dependence of magnetospheric currents and a model of their near-Earth magnetic field, Earth Planets Space, 62, 843-848, doi:10.5047/eps.2010.07.012.

Maus, S. and Weidelt, P. (2004), Separating the magnetospheric disturbance magnetic field into external and transient internal contributions using a 1D conductivity model of the Earth, Geophys. Res. Lett., 31, L12614, doi:10.1029/2004GL020232.

Maus, S. and H. Lühr, Signature of the quiet-time magnetospheric magnetic field and its electromagnetic induction in the rotating Earth, Geophys. J. Int., 162, 755-763, doi:10:1111/j.1365-246X.2005.02691.x, 2005.

Newell, P. T., and J. W. Gjerloev (2012), SuperMAG-based partial ring current indices, J. Geophys. Res., 117, A05215, doi:10.1029/2012JA017586.

Newell, P. T., Sotirelis, T., Liou, K., Meng, C.-I., and Rich, F. J.: A nearly universal solar wind-magnetosphere coupling function inferred from magnetospheric state variables, J. Geophys. Res., 112, A01206, doi:10.1029/2006JA012015, 2007.

Olsen, P.W., The geomagnetic field and its extension into space, Adv. Space Res., 2(1), 13$17,1982$.

Olsen, N., Sabaka, T.J. \& Lowes, F., 2005. New parameterization of external and induced fields in geomagnetic field modeling, and a candidate model for IGRF 2005, Earth, Planets and Space, 57, 1141-1149.

Olsen, N., H. Lühr, C.C. Finlay, T.J. Sabaka, I. Michaelis, J. Rauberg, L. Tøffner-Clausen (2014), The CHAOS-4 Geomagnetic Field Model, Geophys. J. Int., 197, 815-827, doi:10.1093/gji/ggu033.

Ritter, P. and H. Lühr, Near-Earth magnetic signature of magnetospheric substorms and an improved substorm current model, Ann. Geophys., 26, 2781-2793, 2008. 
Sckopke, N. (1966), A general relation between the energy of trapped particles and the disturbance field near the Earth, J. Geophys. Res., 71, 3125-3130.

Shue, J.-H., P. Song, C. T. Russell, J. T. Steinberg, J. K. Chao, G. Zastenker, O. L. Vaisberg, S. Kokubun, H. J. Singer, T. R. Detman, H. Kawano, Magnetopause location under extreme solar wind conditions, J. Geophys. Res., 103(A8), 17691-17700, 1998.

Sibeck, D. G., R. E. Lopez, and E. C. Roelof (1991), Solar wind control of the magnetopause shape, location, and motion, J. Geophys. Res., 96(A4), 5489-5495, doi:10.1029/90JA02464

Sugiura, M.: Hourly values of equatorial Dst for the IGY, Ann. Int. Geophys. Year, 35, 9-45, 1964.

Thomson, A. and Lesur, V. (2007), An improved geomagnetic data selection algorithm for global geomagnetic field modelling, Geophys. J. Int., 169:951-963, doi:10.1111/j.1365-246X.2007.03354.x.

Tsyganenko, N. A., and D. H. Fairfield (2004), Global shape of the magnetotail current sheet as derived from Geotail and Polar data, J. Geophys. Res., 109, A03218, doi:10.1029/2003JA010062.

Xiong, C. and H. Lühr (2014), An empirical model of the auroral oval derived from CHAMP field-aligned current signatures - Part 2, Ann. Geophys., 32, 623-631, 2014, doi:10.5194/angeo-32-623-2014.

Xiong, C., H. Lühr, H. Wang, and M.G. Johnsen (2014), Determining the boundaries of the auroral oval from CHAMP field-aligned currents signatures - Part 1, Ann. Geophys., 32, 609-622, 2014, doi:10.5194/angeo-32-609-2014.

Zhang, Q.-H., M. W. Dunlop, M. Lockwood, R. Holme, Y. Kamide, W. Baumjohann, R.-Y. Liu, H.-G. Yang, E. E. Woodfield, H.-Q. Hu, B.-C. Zhang, and S.-L. Liu (2011), The distribution of the ring current: Cluster observations, Ann. Geophys., 29, 1655-1662, doi:10.5194/angeo-29-1655-2011. 